\title{
Infectious Diseases and the Kidney in Children
}

\author{
Jennifer Stevens $^{\mathrm{a} *}$, Jethro A. Herberg ${ }^{\mathrm{b}}$ and Michael Levin ${ }^{\mathrm{c}}$ \\ ${ }^{a}$ University Hospital Wales, Cardiff, S. Wales, UK \\ ${ }^{\mathrm{b}}$ Imperial College London, London, UK \\ ${ }^{\mathrm{c}}$ Department of Medicine, Imperial College London, London, UK
}

The kidney is involved in a wide range of bacterial, viral, fungal, and parasitic diseases. In most systemic infections, renal involvement is a minor component of the illness, but in some, renal failure may be the presenting feature and the major problem in management. Although individual infectious processes may have a predilection to involve the renal vasculature, glomeruli, interstitium, or collecting systems, a purely anatomic approach to the classification of infectious diseases affecting the kidney is rarely helpful because most infections may involve several different aspects of renal function. In this chapter, a microbiological classification of the organisms affecting the kidney is adopted. Although they are important causes of renal dysfunction in infectious diseases, urinary tract infections and hemolytic uremic syndrome (HUS) are not discussed in detail because they are considered separately in chapters $>$ Renal Involvement in Children with HUS and $>$ Urinary Tract Infections in Children, respectively.

Elucidation of the cause of renal involvement in a child with evidence of infection must be based on a careful consideration of the geographic distribution of infectious diseases in different countries. A history of foreign travel; exposure to animals, insects, or unusual foods or drinks; outdoor activities such as swimming or hiking; and contact with infectious diseases must be sought in every case. The clinical examination should include a careful assessment of the skin and mucous membranes and a search for insect bites, lymphadenopathy, and involvement of other organs. A close collaboration with a pediatric infectious disease specialist and hospital microbiologist will aid the diagnosis and management of the underlying infection.

A tantalizing clue to the pathogenesis of glomerular disease is the marked difference in the incidence of nephrosis and nephritis in developed and underdeveloped areas of the world. In several tropical countries, glomerulonephritis (GN) accounts for up to $4 \%$ of pediatric hospital admissions; the incidence in temperate climates is 10 - to 100 -fold less. This difference might be explained by a complex interaction of several different factors, including nutrition, racial and genetically determined differences in immune responses, and exposure to infectious diseases. A growing body of evidence, however, suggests that long-term exposure to infectious agents is a major factor in the increased prevalence of glomerular diseases in developing countries.

Renal involvement in infectious diseases may occur by a variety of mechanisms: direct microbial invasion of the renal tissues or collecting system may take place in conditions such as staphylococcal abscess of the kidney occurring as a result of septicemic spread of the organism; ascending infection commonly occurs due to infection in the urinary tract; damage to the kidney may be caused by the systemic release of endotoxin or other toxins and activation of the inflammatory cascade during septicemia or due to a focus of infection distant from the kidney; ischemic damage may result from inadequate perfusion induced by septic shock; the kidney may be damaged by activation of the

*Email: stevensjae@hotmail.com 
immunologic pathways or by immune complexes resulting from the infectious process. In many conditions, a combination of these mechanisms may be operative. In the assessment of renal complications occurring in infectious diseases, the possibility of drug-induced nephrotoxicity caused by antimicrobial therapy should always be considered. The nephrotoxic effects of antibiotics and other antimicrobial agents are not addressed in this chapter but are covered in chapter XX.

\section{Bacterial Infections}

Bacterial infections associated with renal disease and the likely mechanisms causing renal dysfunction are shown in Table 1.

\section{Systemic Sepsis and Septic Shock}

Impaired renal function is common in systemic sepsis, and acute kidney injury (AKI) is an independent risk factor for mortality in pediatric sepsis [1]. Depending on the severity of the infection and the organism responsible, the renal involvement may vary from insignificant proteinuria to AKI requiring dialysis. The organisms causing AKI as part of systemic sepsis vary with age and geographic location and also differ in normal and immunocompromised children. In the neonatal period, group B streptococci, coliforms, Staphylococcus aureus, and Listeria monocytogenes are the organisms usually responsible. In older children, Neisseria meningitidis, Streptococcus pneumoniae, group A Streptococcus, and Staphylococcus aureus account for most of the infections. In people who are immunocompromised, a wide range of bacteria are seen, and, similarly, in tropical countries, other pathogens, including Haemophilus influenzae, Salmonella species, and Pseudomonas pseudomallei, must be considered. Vaccines against $N$. meningitides, $S$. pneumoniae, and $H$. influenzae have reduced the impact of these infections, but their uptake varies in different countries.

Systemic sepsis usually presents with nonspecific features: fever, tachypnea, tachycardia, and evidence of skin and organ underperfusion. The pathophysiology of renal involvement in systemic sepsis is multifactorial $[2,3]$. Hypovolemia with diminished renal perfusion is the earliest event and is a consequence of the increased vascular permeability and loss of plasma from the intravascular space. Hypovolemia commonly coexists with depressed myocardial function because of the myocardial depressant effects of endotoxin or other toxins. The renal vasoconstrictor response to diminished circulating volume and reduced cardiac output further reduces glomerular filtration, and oliguria is thus a consistent and early event in severe sepsis. A number of vasodilator pathways are activated in sepsis, including nitric oxide and the kinin pathways. This may lead to inappropriate dilatation of vascular beds. Vasodilatation of capillary beds leading to warm shock is common in adults with sepsis due to Gram-negative organisms but is less commonly seen in children, in whom intense vasoconstriction is the usual response to sepsis. If renal underperfusion and vasoconstriction are persistent and severe, the reversible prerenal failure is followed by established renal failure with the characteristic features of vasomotor nephropathy or acute tubular necrosis.

Other mechanisms of renal damage in systemic sepsis include direct effects of endotoxin and other toxins on the kidney and release of inflammatory mediators such as tumor necrosis factor (TNF) and other cytokines, arachidonic acid metabolites, and proteolytic enzymes. Leukocyteendothelial interactions result in physical congestion of the medullary vasculature and further decrease regional blood flow. Nitric oxide (NO) is postulated to play a key role in the pathophysiology of renal failure in sepsis. NO is produced from three cell-specific nitric oxide synthase (NOS) isoforms. Within the kidney, endothelial NOS (eNOS) is expressed in endothelial cells and plays a 


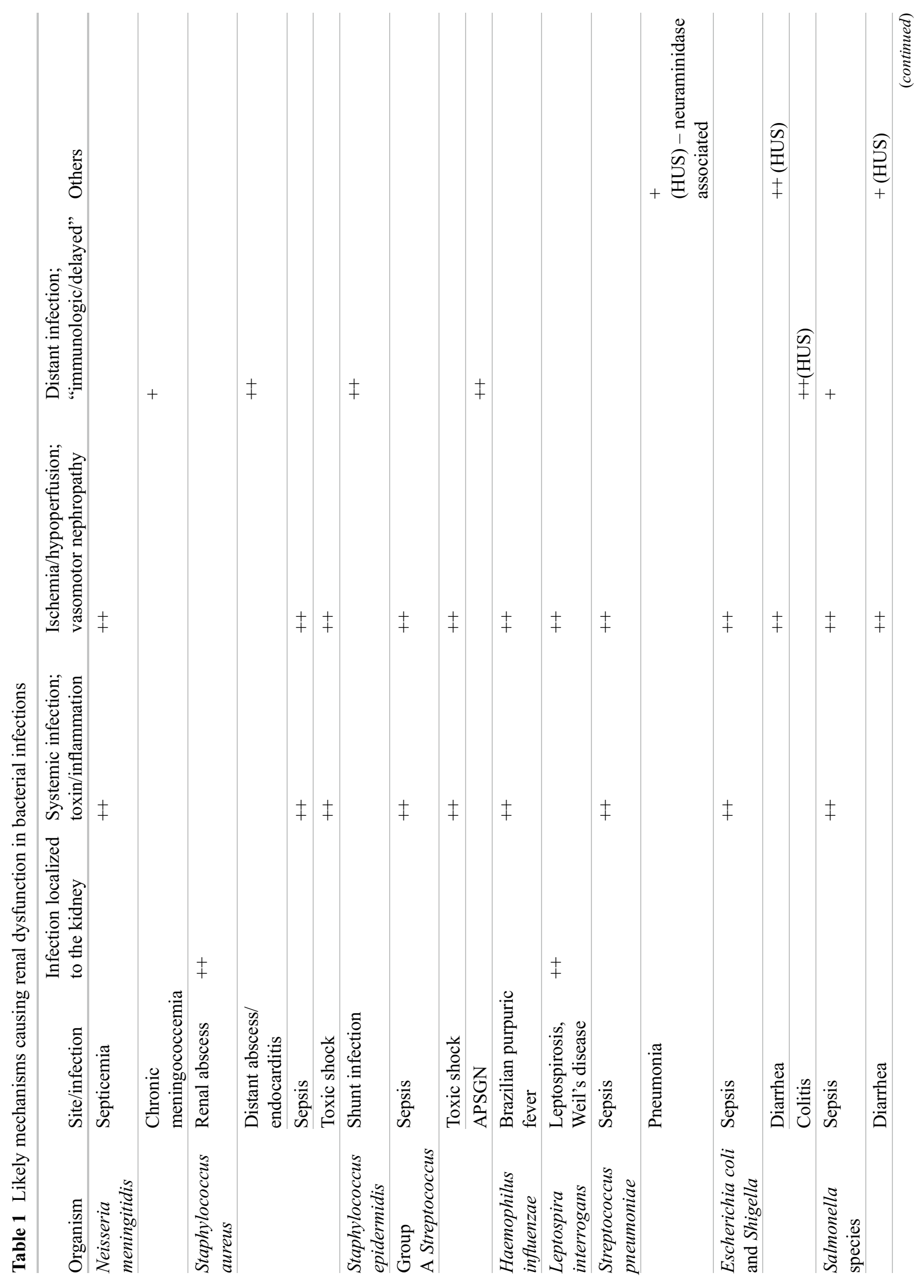




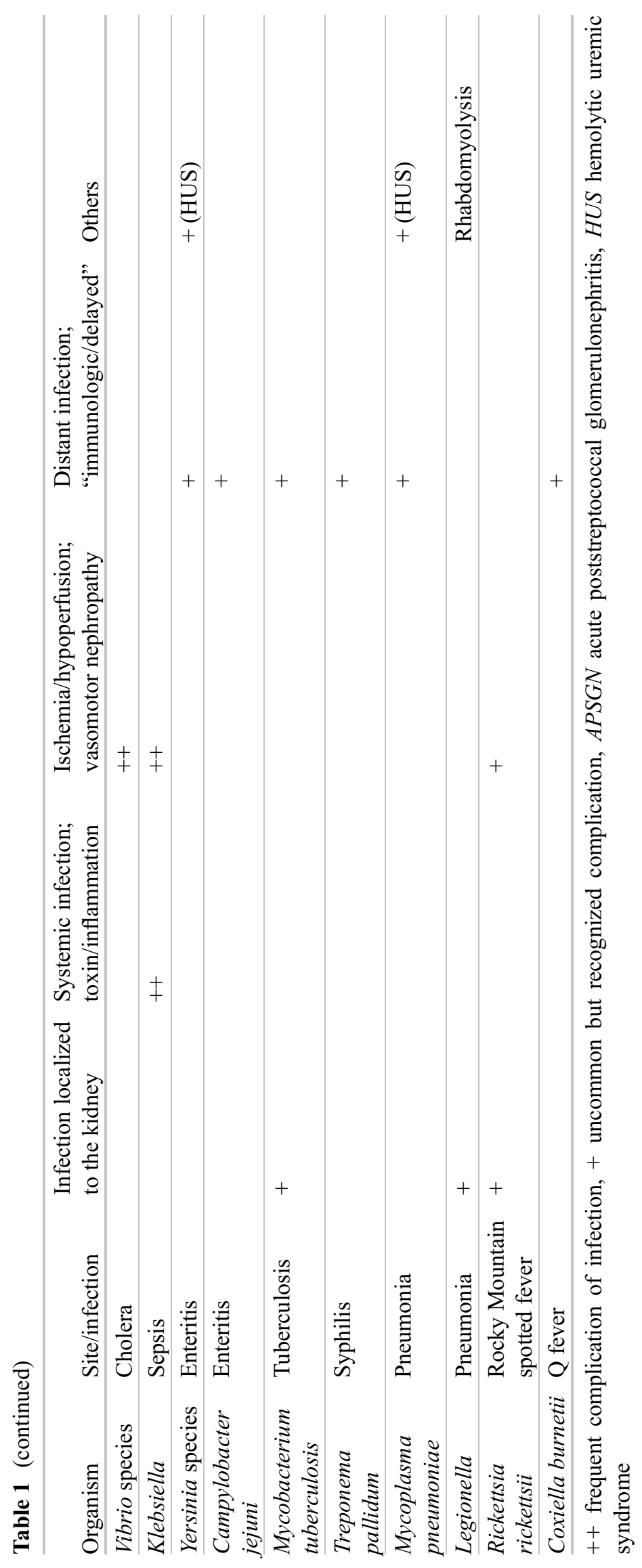


role in vascular relaxation, inhibition of leukocyte adhesion, and platelet aggregation. Endothelial injury in renal ischemia has been reported to impair the production of NO by eNOS. Inducible NOS (iNOS) has been implicated as an important mediator of vasodilatation and is upregulated within the medulla and the glomeruli in sepsis. The alteration of NOS expression and NO production within the systemic circulation and the kidney has supported the extensive testing of NOS inhibitors in sepsis and renal ischemia [4]. Trials of selective NO synthase inhibition did not offer any advantages over saline resuscitation [5]. Activation of coagulation is an important component of the pathophysiology of septic shock and contributes to intraglomerular thrombosis. Tubular injury then leads to cell detachment and intratubular obstruction and tubular backleak. Recovery necessitates the clearance of the necrotic cells and debris as well as the repair of the nonfatally injured cells. Activation of multiple prothrombotic and antifibrinolytic pathways occurs, together with downregulation of antithrombotic mechanisms such as the protein $\mathrm{C}$ pathway. Treatment with activated protein $\mathrm{C}$ has been shown to improve outcome of adult septic shock, but has not been confirmed to have benefit in pediatric sepsis and may carry a risk of bleeding particularly in infants [6].

The renal findings early in septic shock are oliguria, with high urine/plasma urea and creatinine ratios, low urine sodium concentration, and a high urine/plasma osmolarity ratio. Once established, renal failure supervenes, and the urine is of poor quality with low urine/plasma urea and creatinine ratios, elevated urine sodium concentration, and low urine osmolarity. Proteinuria is usually present, and the urine sediment may contain red cells and small numbers of white cells.

\section{Management of AKI in Systemic Sepsis}

The management of AKI in systemic sepsis depends on early diagnosis and administration of appropriate antibiotics to cover the expected pathogens. Reliance on creatinine and urine output to identify AKI (using, e.g., the pediatric RIFLE score [7]) may in the future be improved using alternative biomarkers, such as neutrophil gelatinase-associated lipocalin (NGAL) [2, 8]. Management is directed at improving renal perfusion and oxygenation. Volume replacement with crystalloid or colloid should be undertaken to optimize preload. Central venous pressure or pulmonary wedge pressure monitoring is essential to guide volume replacement in children in severe shock. The use of low-dose (2-5 pg/kg/min) dopamine to reduce renal vasoconstriction together with administration of inotropic agents such as dobutamine or epinephrine to improve cardiac output may reverse prerenal failure. Early elective ventilation should be undertaken in patients with severe shock. If oliguria persists despite volume replacement and inotropic therapy, dialysis or hemofiltration should be instituted early, because septic and catabolic patients may rapidly develop hyperkalemia and severe electrolyte imbalance.

In most children who develop AKI as part of systemic sepsis or septic shock, the renal failure is of short duration, and recovery can be expected within a few days of achieving cardiovascular stability and eradication of the underlying infection. Occasionally, renal cortical necrosis or infarction of the kidney may result in prolonged or permanent loss of renal function.

\section{Specific Bacterial Infections Causing Renal Disease}

\section{Meningococcus}

Neisseria meningitidis continues to be a major cause of systemic sepsis and meningitis in both developed and underdeveloped parts of the world. In developed countries, most cases are caused by group $\mathrm{B}$ and $\mathrm{Y}$ strains, particularly after introduction of meningococcal $\mathrm{C}$ vaccination, whereas epidemics of meningococcal groups A, C, and W135 continue to occur in many underdeveloped 
regions of the world [9]. In 2013, a meningococcal B vaccine was approved for use in Europe, Canada, and Australia, and it has been used in the United States to help control outbreaks (see www. meningitis.org/menb-vaccine). Peak incidence is in infants for group B disease and teenagers and young adults for groups $\mathrm{C}$. However, the disease can occur at any age. There are two major presentations of meningococcal disease: meningococcal meningitis presents with features indistinguishable from those of other forms of meningitis, including headache, stiff neck, and photophobia. Lumbar puncture is required to identify the causative agent and distinguish this from other forms of meningitis. Despite the acute nature of the illness, the prognosis is good, and most patients with the purely meningitic form of the illness recover without sequelae. The most common sequelae is hearing loss and so all children should have a hearing test after discharge [10].

Meningococcemia with purpuric rash and shock is the second and more devastating form of the illness. Affected patients present with nonspecific symptoms of fever, vomiting, abdominal pain, and muscle ache. The diagnosis is only obvious once the characteristic petechial or purpuric rash appears. Patients with a rapidly progressive purpuric rash, hypotension, and evidence of skin and organ underperfusion have a poor prognosis, with a mortality of 10-30\%. Adverse prognostic features include hypotension, a low white cell count, absence of meningeal inflammation, thrombocytopenia, and disturbed coagulation indices or a combination of these [11].

Renal failure was seldom reported in early series of patients with meningococcemia, perhaps because most patients died rapidly of uncontrolled septic shock. With advances in intensive care, however, more children are surviving the initial period of profound hemodynamic derangement, and renal failure is more often seen as a major management problem. Children with fulminant sepsis, particularly with Gram-negative organisms including N. meningitidis, can develop renal failure in association with rhabdomyolysis [12].

The pathophysiology of meningococcal septicemia involves the activation of cytokines and inflammatory cells by endotoxin [13]. Mortality is directly related to both the plasma endotoxin concentration and the intensity of the inflammatory response, as indicated by levels of TNF and other inflammatory markers. Patients with meningococcemia have a profound capillary leak leading to severe hypovolemia. A loss of plasma proteins from the intravascular space is probably the major cause of shock, but myocardial suppression secondary to IL6 production is also important [14]. Intense vasoconstriction further impairs tissue and organ perfusion, and vasculitis with intravascular thrombosis and consumption of platelets and coagulation factors is also present.

Oliguria is invariably present in children with meningococcemia during the initial phase of the disorder. This is prerenal in origin and may respond to volume replacement and inotropic support. If cardiac output cannot be improved and renal underperfusion persists, established renal failure supervenes. Occasionally, cortical necrosis or infarction of the kidneys occurs. Children with meningococcemia should be aggressively managed in a pediatric intensive care unit, with early administration of antibiotics (penicillin or a third-generation cephalosporin), volume replacement, hemodynamic monitoring, and the use of inotropic agents and vasodilators. If oliguria persists despite measures to improve cardiac output, elective ventilation and dialysis should be instituted early. Because activation of coagulation pathways occurs, severe acquired protein $\mathrm{C}$ deficiency may result and is usually associated with substantial mortality [15]. Protein $\mathrm{C}$ is a natural anticoagulant which also has an important anti-inflammatory activity. Despite evidence for impaired function of the activated protein $C$ pathway in meningococcal diseases [15] and adult trials suggesting benefit of activated protein $\mathrm{C}$ administration in septic shock (PROWESS trial) [16], pediatric trials of activated protein $\mathrm{C}$ showed no clear benefit and were associated with increased risk of intracranial bleeding in very young infants [6]. The role of aPC therapy in pediatric sepsis remains unclear. Most patients 
who survive the initial $24-48 \mathrm{~h}$ of the illness and regain hemodynamic stability will ultimately recover renal function even if dialysis is required for several weeks.

The least common presentation of meningococcal sepsis is chronic meningococcemia. Patients with this form of the illness present insidiously with a vasculitic rash, arthritis, and evidence of multiorgan involvement. The features may overlap those of Henoch-Schonlein purpura or subacute bacterial endocarditis (SBE), and the diagnosis must be considered in patients presenting with fever, arthritis, and vasculitic rash, often accompanied by proteinuria or hematuria. Response to antibiotic treatment is good, but some patients may have persistent symptoms for many days resulting from an immune-complex vasculitis.

\section{Staphylococcus Aureus}

Staphylococcal infections may affect the kidneys by direct focal invasion during staphylococcal septicemia, forming a renal abscess, by causing staphylococcal bacteremia, or by toxin-mediated mechanisms, as in the staphylococcal toxic shock syndrome.

Staphylococcal Abscess. Staphylococcal renal abscess presents with fever, loin pain and tenderness, and abnormal urine sediment, as do abscesses caused by other organisms. The illness often follows either septicemia or pyelonephritis. The diagnosis is usually considered only when a patient with clinical pyelonephritis shows an inadequate response to antibiotic treatment. The diagnosis is confirmed by ultrasonography or computed tomographic scan, which shows swelling of the kidney and intrarenal collections of fluid. Antibiotic therapy alone may result in cure, and empirical therapy should cover both Gram-positive and Gram-negative organisms until microbiological diagnosis has been achieved. Treatment may require percutaneous drainage alongside antibiotic therapy, which may need to persist for 2-4 weeks. Open drainage may be necessary if this approach has failed [17].

Staphylococcal Toxic Shock Syndrome. The staphylococcal toxic shock syndrome is a systemic illness characterized by fever, shock, erythematous rash, diarrhea, confusion, and renal failure. The disorder was first described by Todd et al. in 1978 in a series of seven children [18]. During the 1980 s, thousands of cases were reported in the United States. Most cases were in menstruating women in association with tampon use. Menstrual and non-menstrual cases are now equally common, including children of both sexes and all ages [19].

The illness usually begins suddenly with high fever, diarrhea, confusion, and hypotension, together with a diffuse erythroderma [20]. Mucous membrane involvement with hyperemia and ulceration of the lips and oral mucosa or vaginal mucosa, strawberry tongue, and conjunctival injection are usually seen. Desquamation of the rash occurs in the convalescent phase of the illness. Confusion is often present in the early stages of the illness and may progress to coma in severe cases. Multiple organ failure with evidence of impaired renal function, elevated levels of hepatic transaminases, thrombocytopenia, elevated CPK, and disseminated intravascular coagulation (DIC) is often seen.

According to CDC criteria, the diagnosis is made on the basis of the clinical features of fever, rash, hypotension, and subsequent desquamation along with deranged function of three or more of the following organ systems: the gastrointestinal (GI), mucous membranes, renal, hepatic, hematologic, central nervous system, and muscle. Other disorders causing a similar picture, such as Rocky Mountain spotted fever, leptospirosis, measles, and streptococcal infection, must be excluded.

The staphylococcal toxic shock syndrome is caused by infection or colonization with strains of $S$. aureus that produce one or more protein exotoxins [21]. Most cases in adults are associated with toxic shock toxin I; in children, many of the isolates associated with the syndrome produce other enterotoxins (A to F). The staphylococcal enterotoxins induce disease by acting as superantigens [22], which activate $\mathrm{T}$ cells bearing specific $\mathrm{V}$ beta regions of the T-cell receptor; this causes 
proliferation and cytokine release. The systemic illness and toxicity are believed to result largely from an intense inflammatory response induced by the toxin. The site of toxin production is often a trivial focus of infection or simple colonization, and bacteremia is rarely observed.

Renal failure in toxic shock syndrome is usually caused by shock and renal hypoperfusion. In the early stages of the illness, oliguria and renal impairment are usually prerenal and respond to treatment of shock and measures to improve perfusion. In severe cases and in patients in whom treatment is delayed, AKI develops as a consequence of prolonged renal underperfusion, and dialysis may be required. In addition to underperfusion, direct effects of the toxin or inflammatory mediators may also contribute to the renal damage. Recovery of renal function usually occurs, but in severe cases with cortical necrosis or intense renal vasculitis, prolonged dialysis may be required.

The management of staphylococcal toxic shock syndrome depends on early diagnosis and aggressive cardiovascular support with volume replacement, inotropic support, and, in severe cases, elective ventilation. If oliguria persists despite optimization of intravascular volume and administration of inotropic agents, dialysis should be commenced early [19].

Antistaphylococcal antibiotics should be started as soon as the diagnosis is suspected and the site of infection identified. Current advice in the Red Book (http://aapredbook.aappublications.org) is that initial empiric antimicrobial therapy should include an antistaphylococcal antibiotic effective against beta-lactamase-resistant organisms and a protein synthesis-inhibiting antibiotic such as clindamycin to stop further toxin production [23]. If there is a focus of infection such as a vaginal tampon, surgical wound, or infected sinuses, the site should be drained early to prevent continued toxin release into the circulation. The intravenous administration of immunoglobulins may be considered when infection is refractory to several hours of aggressive therapy, an undrainable focus is present, or persistent oliguria with pulmonary edema occurs. With aggressive intensive care, most affected patients survive, and renal recovery is usual, even in patients who have had severe shock and multiorgan failure. Relapses and recurrences of staphylococcal toxic shock syndrome occur in a proportion of affected patients because immune responses to the toxin are ineffective in some individuals.

Panton-Valentine Leukocidin (PVL)-Producing Staphylococcal Infection. In recent years, there have been increasing reports of severe staphylococcal disease, associated with shock and multiorgan failure, caused by strains of staphylococci producing the PVL toxin. Panton-Valentine leukocidin (PVL) is a phage-encoded toxin, which profoundly impairs the host response due to its toxic effect on leukocytes (see review [24]). PVL-producing strains are associated with tissue necrosis and increased propensity to cause abscesses in lung, bone, joint, and soft tissue infections. Perinephric abscesses have been reported [25]. There are increasing numbers of children and adults admitted with fulminant sepsis and shock due to PVL-producing strains, and renal failure is a significant component of the multiorgan failure. In addition to intensive care support, antibiotic treatment of PVL strains should include antibiotics which reduce toxin production, such as clindamycin, linezolid or rifampicin, as well as vancomycin if the strain is resistant to methicillin. Beta-lactam antibiotics should be avoided, as there is some data to suggest that PVL toxin production can be increased by these antibiotics under some conditions [23, 26]. Immunoglobulin infusion may also be of benefit. PVL sepsis is often associated with thrombosis, and prophylactic heparin should be commenced in seriously ill patients. Aggressive surgical drainage of all collections requires close consultation with orthopedic and surgical teams.

\section{Streptococcus Pyogenes}

The group A streptococci (GAS) are extracellular Gram-positive organisms. They are a major worldwide cause of renal disease, usually as poststreptococcal nephritis. However, in addition to 
this postinfection immunologically mediated disorder, GAS can cause AKI as part of an invasive infection with many features of the staphylococcal toxic shock syndrome.

Acute Poststreptococcal Glomerulonephritis. Acute poststreptococcal GN (APSGN) is a delayed complication of pharyngeal infection or impetigo with certain nephritogenic strains of GAS, though many cases of postinfectious GN are unrelated to Streptococcus [27]. Worldwide, the incidence of APSGN is decreasing, although clusters of cases continue to occur [28]. APSGN after pharyngeal infection is associated with different serotypes as compared to pyoderma and skin infections [28]. Different strains can be serotyped according to the antigenic properties of the $M$ protein found in the outer portion of the bacterial wall. The pathology and pathogenesis of the disorder is discussed in detail in chapter XX.

Detection of GAS in patients with APSGN may be possible by culture from the skin or the throat in some patients. Other evidence of infection with a GAS can be obtained through the antistreptolysin-O titer (ASOT), which is increased in $60-80 \%$ of cases. Early antibiotic treatment can reduce the proportion of cases with elevated ASOT to $30 \%$. Anti-deoxyribonuclease B and antihyaluronidase testing has been shown to be of more value than ASOT in confirming group A streptococcal infection in impetigo-associated cases. Decreased C3 and total hemolytic complement levels are found in $90 \%$ of cases during the first 2 weeks of illness and return to normal after 4-6 weeks.

Penicillin should be given to eradicate the GAS organisms. Erythromycin, clindamycin, or a firstgeneration cephalosporin can be given to patients allergic to penicillin. Early antibiotic therapy can help to prevent the immune response against the streptococcal antigens and thus the progression to glomerulonephritis and other rheumatic fever sequelae. Treatment failures have been thought to be due to the coexistence of beta-lactamase-producing bacteria in the tonsillopharynx or due to streptococci that have invaded the epithelial cells and are protected from the antibiotics. In these instances, amoxicillin was given with clavulanate [29]. Close contacts and family members who are culture-positive for GAS should also be given penicillin, although antibiotic treatment is not always effective in eliminating secondary cases. Recurrent episodes are rare, and immunity to the particular nephritogenic strain that caused the disease is probably lifelong. Antibiotic prophylaxis is therefore unnecessary.

Most studies suggest that the prognosis for children with APSGN is good, with more than $90 \%$ making a complete recovery. However, $10 \%$ of cases may have a prolonged and more serious course with long-term chronic renal failure [30].

Other Streptococci. APSGN has also been described after outbreaks of group C streptococcal infection. For example, Streptococcus equi subsp. zooepidemicus has been found responsible for large epidemics in South America [31]. Group C streptococcal infection has occurred after consumption of unpasteurized milk from cattle with mastitis. Patients developed pharyngitis followed by APSGN. Endostreptosin was found in the cytoplasm of these group C strains, and during the course of the illness, patients developed anti-endostreptosin antibodies. This antigen has been postulated to be the nephritogenic component of GAS.

In addition, strains of group $\mathrm{G}$ streptococci have been implicated in occasional cases of APSGN [32]. Isolates possessed the type M12 protein antigen identical to the nephritogenic type M12 antigen of some group A streptococcal strains.

Streptococcal Toxic Shock Syndrome and Invasive Group A Streptococcal Infection. Group A Streptococcus causes a severe illness with similarities to the staphylococcal toxic shock syndrome, occurring in both children and adults, associated with invasive group A streptococcal disease [33]. Patients with this syndrome present acutely with high fever, erythematous rash, mucous membrane involvement, hypotension, and multiorgan failure [34, 35]. Unlike staphylococcal 
toxic shock syndrome, in which the focus of infection is usually trivial and bacteremia is seldom seen, the streptococcal toxic shock syndrome is usually associated with bacteremia or a serious focus of infection such as septic arthritis, myositis, or osteomyelitis. Laboratory findings of anemia, neutrophil leukocytosis, thrombocytopenia, and DIC are often present, together with impaired renal function, hepatic derangement, and acidosis. AKI requiring dialysis occurs in a significant proportion of cases.

It is not clear why there are increasing numbers of cases with invasive disease caused by GAS, nor why there has been an emergence of streptococcal toxic shock syndrome and indeed a similar syndrome caused by some Pseudomonas and Klebsiella strains. The most common antecedent of invasive GAS disease is varicella infection, with the streptococcal infection developing after the initial vesicular phase of the disease is subsiding. Strains causing toxic shock syndrome and invasive disease appear to differ from common isolates of GAS in producing large amounts of pyrogenic toxins that may have superantigen-like activity. Another important mechanism is the production by invasive GAS of an IL8 protease. IL8 serves as a molecular bridge between receptors on neutrophils and the vascular endothelium. Cleavage of this protein prevents neutrophil attachment to the endothelium and results in uncontrolled spread of the bacteria through the tissues [36]. In severe cases, necrotizing fasciitis occurs with extensive destruction of the subcutaneous tissues and is often associated with multiorgan failure. The pathophysiology of streptococcal toxic shock syndrome and that of invasive disease is similar in that superantigen toxins that induce release of cytokines and other inflammatory mediators play a role in both conditions. However, GAS toxic shock is usually more severe, carries a higher mortality, and is more often associated with focal collections or necrotizing fasciitis.

Treatment of streptococcal toxic shock syndrome depends on the administration of appropriate antibiotics, aggressive circulatory support, and treatment of any multiorgan failure. Surgical intervention to drain the infective focus in the muscle, bone, joint, or body cavities is often required. Antibiotic therapy with beta-lactams should be supplemented by treatment with a protein synthesisinhibiting antibiotic, such as clindamycin, and it is suggested that this limits new toxin production $[37,38]$.

Pooled intravenous immunoglobulins are now in widespread use in the treatment of toxic shock, particularly when caused by Streptococcus [39, 40], though not all studies have reported benefit [35]. Prospective observational studies have suggested that the concomitant use of immunoglobulin with clindamycin has a positive synergistic effect on mortality [41, 42]. The role of steroids remains unclear, with their hemodynamic benefit set against the detrimental effects of hyperglycemia secondary to gluconeogenesis $[43,44]$. The benefit of insulin therapy to control hyperglycemia is unclear. A study in adults found that intensive insulin therapy increased the risk of serious adverse events [45]. In contrast to adult patients, in children with severe sepsis, the use of activated protein $\mathrm{C}$ (drotrecogin) cannot be recommended, as in a multicenter trial, fatality was increased in the treatment group [6]. Recovery of renal function occurs in patients who respond to treatment of shock and the eradication of the infection.

\section{Streptococcus Pneumoniae}

Infection with $S$. pneumoniae is one of the most common infections in humans and causes a wide spectrum of disease, including pneumonia, otitis media, sinusitis, septicemia, and meningitis. Despite the prevalence of the organism, a significant renal involvement is relatively rare but is seen in two situations: pneumococcal septicemia in asplenic individuals or in those with other immunodeficiencies presents with fulminant septic shock in which renal failure may occur as part of 
a multisystem derangement. The mortality from pneumococcal sepsis in asplenic patients is high, even with early antibiotic treatment and intensive support.

The second nephrologic syndrome associated with $S$. pneumoniae is a rare form of HUS. It accounts for 5-15\% of all HUS cases in children and typically develops 3-13 days after streptococcal infection. The incidence following streptococcal infection is $0.4-0.6 \%$. Since the introduction of the 7- and 13-valent vaccines, there have been reports of HUS cases caused by strain not covered in the vaccine [46]. In 1955, Gasser and colleagues described HUS as a clinical entity in children, and they included two infants with pneumonia among the five patients they described [47]. HUS associated with pneumococcal infection differs in pathophysiology from that caused by the commoner Vero toxin-producing E. coli. S. pneumoniae (as well as influenza and Clostridia species) produce the enzyme neuraminidase which cleaves sialic acid from the surfaces of exposed cells [48]. Neuraminidase causes desialation of red blood cells, and possibly other blood cells and endothelium, by the removal of terminal neuraminic acid, which leads to unmasking of the Thomsen-Friedenreich antigen ( $\mathrm{T}$ antigen) which is present on the surface of red blood cells, platelets, and glomerular capillary endothelia. Normal serum contains antibodies to this antigen which cause a widespread agglutination of blood cells and platelets when the antigen is exposed, resulting in intravascular obstruction, hemolysis, thrombocytopenia, and renal failure. The direct Coombs test is positive in $90 \%$ of cases of streptococcal HUS [49], either from bound anti-T IgM or from anti-T antibodies. The diagnosis of Thomsen-Friedenreich antibody-induced HUS should be suspected in patients with AKI, thrombocytopenia, and hemolysis after an episode of pneumonia or bacteremia caused by $S$. pneumoniae. Fragmented red blood cells will usually be present on blood film.

Association of HUS with $S$. pneumoniae is defined by culture of pneumococci from a normally sterile site within a week before or after onset of signs of HUS. Clues to a pneumococcal cause, in addition to culture results, include severe clinical disease, especially pneumonia, empyema, pleural effusion, or meningitis; hemolytic anemia; positive results on a direct Coombs test; and difficulties in $\mathrm{ABO}$ crossmatching or a positive minor crossmatch incompatibility [46]. However, when renal disease is seen in the context of severe pneumococcal infection, it is important to maintain a broad diagnostic perspective, because of the frequency of acute tubular necrosis in septic shock and DIC.

Therapy for this syndrome should be with supportive treatment and antibiotics (usually a thirdgeneration cephalosporin); dialysis may be required if renal failure occurs. Because normal serum contains antibodies against the Thomsen-Friedenreich antigen, blood transfusion should be undertaken with washed red blood cells resuspended in albumin rather than plasma [50]. Exchange transfusion and plasmapheresis have been used in some patients, with the rationale that these procedures may improve outcome by eliminating circulating neuraminidase [51]. Intravenous IgG has been used in a patient and was shown to neutralize neuraminidase present in the patient's serum [52].

In comparison to patients with the more common diarrhea-associated HUS, S. pneumoniae-induced HUS patients have a more severe renal disease. They are more likely to require dialysis. Their long-term outcome may be affected by the severity of the invasive streptococcal disease itself, and a significant proportion of surviving patients (30-70\%) develop end-stage renal failure [53]. A review of UK cases found an eightfold increase in early mortality as compared to diarrhea-induced HUS. Fresh frozen plasma should be avoided unless there is active bleeding as there are concerns that the preformed anti-TF antibodies are present in FFP [51]. 


\section{Leptospira}

Leptospirosis is an acute generalized infectious disease caused by spirochetes of the genus Leptospira. It is primarily a disease of wild and domestic animals, and humans are infected only occasionally through contact with animals. Most human cases occur in summer or autumn and are associated with exposure to Leptospira-contaminated water or soil during recreational activities such as swimming or camping. In adolescents and adults, occupational exposure through farming or other contact with animals is the route of infection.

The spirochete penetrates intact mucous membranes or abraded skin and disseminates to all parts of the body, including the cerebrospinal fluid (CSF). Although Leptospira do not contain classic endotoxins, the pathophysiology of the disorder has many similarities to that of endotoxemia. In severe cases, jaundice occurs because of hepatocellular dysfunction and cholestasis. Renal functional abnormalities may be profound and out of proportion to the histologic changes in the kidney [54]. Renal involvement is predominantly a result of tubular damage, and spirochetes are commonly seen in the tubular lesions. The inflammatory changes in the kidney may result from either a direct toxic effect of the organism or immune-complex nephritis. However, hypovolemia, hypotension, and reduced cardiac output caused by myocarditis may contribute to the development of renal failure. In severe cases, a hemorrhagic disorder caused by widespread vasculitis and capillary injury also occurs [54, 55].

The clinical manifestations of leptospirosis are variable. Of affected patients, $90 \%$ have the milder anicteric form of the disorder, and only 5-10\% have severe leptospirosis with jaundice. The illness may follow a biphasic course. After an incubation period of 7-12 days, a nonspecific flulike illness lasting 4-7 days occurs, associated with septicemic spread of the spirochete. The fever then subsides, only to recur for the second, "immune," phase of the illness. During this phase, the fever is low grade, and there may be headache and delirium caused by meningeal involvement, as well as intense muscular aching. Nausea and vomiting are common. Examination usually reveals conjunctival suffusion, erythematous rash, lymphadenopathy, and meningism.

The severe form of the disease (Weil's disease) presents with fever, impaired renal and hepatic function, hemorrhage, vascular collapse, and altered consciousness. In one series, the most common organs involved were the liver (71\%) and kidney (63\%). Cardiovascular (31\%), pulmonary (26\%), neurologic (5\%), and hematologic (21\%) involvements were less common [56]. Vasculitis, thrombocytopenia, and uremia are considered important factors in the pathogenesis of hemorrhagic disturbances and the main cause of death in severe leptospirosis [57]. Urinalysis results are abnormal during the leptospiremic phase with proteinuria, hematuria, and casts. Uremia usually appears in the second week, and AKI may develop once cardiovascular collapse and DIC are present [58].

The clinical features of leptospirosis overlap with those of several other acute infectious diseases, including Rocky Mountain spotted fever, toxic shock syndrome, and streptococcal sepsis. The diagnosis of leptospirosis should be considered in febrile patients with evidence of renal, hepatic, and mucous membrane changes and rash, particularly if a history of exposure to freshwater is found. Diagnosis can be confirmed by isolation of the spirochetes from blood or CSF in the first 10 days of the illness or from urine in the second week [58]. The organism may be seen in biopsy specimens of the kidney or skin or in the CSF by dark-field microscopy or silver staining. Serologic tests to detect leptospirosis are now sensitive and considerably aid the diagnosis. Immunoglobulin M (IgM) antibody may be detected as early as 6-10 days into the illness, and antibody titers rise progressively over the next 2-4 weeks. Some patients remain seronegative, and negative serologic test results do not completely exclude the diagnosis. In one series, levels of IgM and IgG anticardiolipin concentrations were significantly increased in leptospirosis patients with AKI [57]. Leptospirosis is treated with intravenous penicillin or other beta-lactam antibiotics. The severity of leptospirosis is reduced by antibiotic treatment, even if started late in the course of the illness [55]. Supportive treatment with 
volume replacement to correct hypovolemia, administration of inotropes, and correction of coagulopathy is essential in severe cases. Dialysis may be required in severe cases and may be needed for prolonged periods until recovery occurs.

\section{Gastrointestinal Infections}

The diarrheal diseases caused by Escherichia coli, Salmonella, Shigella, Campylobacter, Vibrio spp., and Yersinia remain important and common bacterial infections of humans. Although improvements in hygiene and living conditions have reduced the incidence of bacterial gastroenteritis in developed countries, these infections remain common in underdeveloped areas of the world, and outbreaks and epidemics continue to occur in both developed and underdeveloped countries. Renal involvement in the enteric infections may result from any of four possible mechanisms.

Severe Diarrhea and Dehydration. Regardless of the causative organism, diarrhea results in hypovolemia, abnormalities of plasma electrolyte composition, and renal underperfusion. If severe dehydration occurs and is persistent, oliguria from prerenal failure is followed by vasomotor nephropathy and established renal failure.

Systemic Sepsis and Endotoxemia. E. coli, Shigella, and Salmonella (particularly Salmonella typhi) may invade the bloodstream and induce septicemia or septic shock. AKI is commonly seen in infants with E. coli sepsis but is also reported with Klebsiella, Salmonella, and Shigella infections. Its pathophysiology and treatment were discussed previously.

Enteric Pathogen-Associated Nephritis. Enteric infections with E. coli, Yersinia, Campylobacter, and Salmonella have been associated with several different forms of GN, including membranoproliferative GN (MPGN), interstitial nephritis, diffuse proliferative GN, and IgA nephropathy.

In typhoid fever, GN ranging from mild asymptomatic proteinuria and hematuria to AKI may occur [59]. Renal biopsy findings show focal proliferation of mesangial cells, hypertrophy of endothelial cells, and congested capillary lumina. Immunofluorescent studies show IgM, IgG, and C3 deposition in the glomeruli, with Salmonella antigens detected within the granular deposits in the mesangial areas. In the IgA nephropathy after typhoid fever, Salmonella vi antigens have been demonstrated within the glomeruli.

Yersinia infection has been reported as a precipitant of GN. Transient proteinuria and hematuria are found in $24 \%$ of patients with acute Yersinia infection and elevated creatinine levels in $10 \%$. Renal biopsy reveals mild mesangial GN or IgA nephropathy. Yersinia antigens, immunoglobulin, and complement have been detected in the glomeruli. Yersinia pseudotuberculosis is well recognized as one of the causes of acute tubulointerstitial nephritis causing AKI, especially in children; patients have histories of drinking untreated water in endemic areas $[60,61]$. The illness begins with the sudden onset of high fever, skin rash, and GI symptoms. Later in the course, periungual desquamation develops, mimicking Kawasaki disease. Elevated erythrocyte sedimentation rate, C-reactive protein level, and thrombocytosis are noticeable, and mild degrees of proteinuria, glycosuria, and sterile pyuria are common. AKI, which typically develops 1-3 weeks after the onset of fever, follows a benign course with complete recovery. Renal biopsy mainly reveals findings of acute tubulointerstitial nephritis. Antibiotic therapy, although recommended, does not alter the clinical course, but reduces the fecal excretion of the organism [62].

Enteric Pathogen-Induced Hemolytic Uremic Syndrome. HUS is characterized by three distinct clinical signs: AKI, thrombocytopenia, and microangiopathic hemolytic anemia. It was first described in 1955 and was associated with infection by Shiga toxin-producing Shigella dysenteriae. A major breakthrough in the search for the cause of HUS occurred in the 1980s when Karmali et al. reported that 11 of 15 children with diarrhea-associated HUS had evidence of infection with a strain of $E$. coli that produced a toxin active on Vero cells [63]. In diarrhea-associated HUS in the United States and most of Europe, E. coli $0157: \mathrm{H} 7$ is the most important of these strains. E. coli $0157: \mathrm{H} 7$ occurs 
naturally in the GI tract of cattle and other animals, and humans become infected through contaminated food products. Most outbreaks have been associated with consumption of undercooked meat, but unpasteurized milk and cider, drinking water, and poorly chlorinated water for recreational use have also been implicated as vehicles for bacterial spread. HUS is discussed in detail in chapter XX.

\section{Mycobacterium Tuberculosis}

The global epidemic of Mycobacterium tuberculosis is persisting. Several factors have contributed to this, including the emergence of the human immunodeficiency virus (HIV) infection epidemic, large influxes of immigrants from countries in which tuberculosis (TB) is common, the emergence of multiple-drug-resistant $M$. tuberculosis, and breakdown of the health services for effective control of TB in various countries. The World Health Organization (WHO) estimated that in 2012 there were 8.6 million incident cases of tuberculosis (TB) in the world, a rate of 122 per 100,000 population. Tuberculosis deaths were estimated at 1.3 million. One-third of the world's population is currently infected with TB, and the estimated lifetime risk of developing disease is 5-10\% (http://www.who. int/mediacentre/factsheets/fs104/en/).

Mycobacteria, both $M$. tuberculosis and atypical mycobacteria, have also emerged as important causes of opportunistic infection in immunocompromised patients undergoing dialysis and in patients undergoing renal transplantation. The possibility of mycobacterial disease must be considered in patients with fever of unknown origin or unexplained disease in the lungs or other organs. Results of the Mantoux test are often negative, and diagnosis depends on maintaining a high index of suspicion and isolating the organism from the infected site.

Primary Progressive TB. Although most infected children successfully contain the infection and enter a state of latency, about $10 \%$ develop progressive primary or postprimary infection, with mycobacteria disseminating to many organs of the body during the lymphohematogenous phase of the disease. Tubercle bacilli can be recovered from the urine in many cases of miliary TB. Hematogenously spread tuberculomata develop in the glomeruli, which results in caseating, sloughing lesions that discharge bacilli into the tubules. In most cases, the renal lesions are asymptomatic and manifest as mycobacteria in the urine or as sterile pyuria.

Tuberculomata in the cortex may calcify and cavitate or may rupture into the pelvis, discharging infective organisms into the tubules, urethra, and bladder. Dysuria, loin pain, hematuria, and pyuria are the presenting features of this complication, but in many cases, the renal involvement is asymptomatic, even when radiologic and pathologic abnormalities are very extensive. Continuing tuberculous bacilluria may cause cystitis with urinary frequency and, in late cases, a contracted bladder. The intravenous urogram is abnormal in most cases. Early findings are pyelonephritis with calyceal blunting and calyceal-interstitial reflux. Later, papillary cavities may be seen, indicating papillary necrosis. Ureteric strictures, focal calcification, hydronephrosis, and cavitation may also be seen. Renal function is usually well preserved, and hypertension is uncommon. In some cases, either the infection itself or reactions to the chemotherapeutic agents may result in renal failure with evidence of an interstitial nephritis [64].

Renal TB. Classic symptomatic renal TB is a late and uncommon complication in children. The average latency period between pulmonary infection with bacillemia and clinical urogenital tuberculosis is 22 years, and renal TB is more commonly seen in adults [65]. Adult studies have shown that $26-75 \%$ of renal TB coexists with active pulmonary TB and 6-10\% of screened sputumpositive pulmonary TB patients have renal involvement.

The diagnosis is established by isolation of mycobacteria from the urine or by the presence of the characteristic clinical and radiographic features in a child with current or previous TB. Renal TB is treated with drug regimens similar to those used for other forms of $\mathrm{TB}$, with isoniazid, rifampicin, 
pyrazinamide, and ethambutol administered initially for 2 months and isoniazid and rifampicin then continued for a further 7-10 months. Late scarring and urinary obstruction may occur in cases with extensive renal involvement, and such patients should be followed by ultrasonography or intravenous urogram. Surgical intervention may be required in the form of stenting, percutaneous nephrostomy, or partial or total nephrectomy [66].

\section{Treponema Pallidum}

Renal involvement in both congenital and acquired syphilis has an estimated occurrence of $0.3 \%$ in patients with secondary syphilis and between $5 \%$ and $16 \%$ in those with congenital syphilis [67]. The most common manifestation of renal disease in congenital syphilis is the nephrotic syndrome, with proteinuria, hypoalbuminemia, and edema. In some patients, hematuria, uremia, and hypertension may be seen. The renal disease is usually associated with other manifestations of congenital syphilis, including hepatosplenomegaly, rash, and mucous membrane findings.

Nephritis in congenital syphilis is usually associated with evidence of complement activation, with depressed levels of Clq, C4, C3, and C5. Histologic findings are a diffuse proliferative GN or a membranous nephropathy. The interstitium shows a cellular infiltrate of polymorphonuclear and mononuclear cells [68]. Immunofluorescent microscopy reveals diffuse granular deposits of IgG and C3 along the glomerular basement membrane (GBM). Mesangial deposits may also contain IgM. On electron microscopy, scattered subepithelial electron-dense deposits are seen, with fusion of epithelial cell foot processes [68].

Good evidence exists that renal disease is due to an immunologically mediated reaction to treponemal antigens. Antibodies reactive against treponemal antigens can be eluted from the glomerular deposits, and treponemal antigens are present in the immune deposits. Treatment of both congenital and acquired syphilis with antibiotics results in rapid improvement in the renal manifestations [68].

\section{Mycoplasma Pneumoniae}

Renal involvement is surprisingly rare in Mycoplasma pneumoniae infection considering the prevalence of this organism and its propensity to trigger immunologically mediated diseases such as erythema multiforme, arthritis, and hemolysis. Acute nephritis associated with Mycoplasma infection may occur 10-40 days after the respiratory tract infection [69]. A few cases of IgA nephropathy have been reported following Mycoplasma infection [70]. Renal histopathologic findings include type $1 \mathrm{MPGN}$, proliferative endocapillary GN, and minimal change disease [71]. Antibiotic treatment of the infection does not appear to affect the renal disease, which is self-limited in most cases [69].

\section{Legionnaires' Disease}

Since its recognition in 1976, Legionnaires' disease, caused by Legionella pneumophila, has emerged as an important cause of pneumonia. The disease most commonly affects the elderly but has been reported in both normal and immunocompromised children. Renal dysfunction occurs in a minority of patients [72]. Those who develop renal failure can present with transient azotemia, hematuria, proteinuria, pyuria, or cylindruria. They are usually severely ill, with bilateral pulmonary infiltrates, fever, and leukocytosis. Shock may be present, and the renal impairment has been associated with acute rhabdomyolysis with high levels of creatine phosphokinase and myoglobinuria. Renal histologic examination usually shows a tubulointerstitial nephritis or acute tubular necrosis $[72,73]$. The pathogenesis of the renal impairment is uncertain, but the organism has been detected within the kidney on electron microscopy and immunofluorescent studies, which 
suggests a direct toxic effect. Myoglobinuria and decreased perfusion may also be contributing factors, however. Mortality has been high in reported cases of Legionnaires' disease complicated by renal failure. Treatment is based on dialysis, intensive care, and antimicrobial therapy with erythromycin [72]. Steroid therapy may be effective for tubulointerstitial nephritis [73].

\section{Rickettsial Diseases}

The rickettsial diseases are caused by a family of microorganisms that have characteristics common to both bacteria and viruses and that cause acute febrile illnesses associated with widespread vasculitis. With the exception of Q fever, all are associated with erythematous rashes. There are four groups of rickettsial diseases:

1. The typhus group includes louse-borne and murine typhus, spread by lice and fleas, respectively.

2. The spotted fever group includes Rocky Mountain spotted fever, tick typhus, and related Mediterranean spotted fever and rickettsial pox, which are spread by ticks and mites, with rodents as the natural reservoir.

3. Scrub typhus, which is spread by mites.

4. Q fever, which is spread by inhalation of infected particles from infected animals.

Rickettsial diseases have a worldwide distribution and vary widely in severity, from self-limited infections to fulminant and often fatal illnesses. In view of the widespread vasculitis associated with these infections, subclinical renal involvement probably occurs in many of the rickettsial diseases. However, in Rocky Mountain spotted fever, tick typhus, and Q fever, the renal involvement may be an important component of the illness.

\section{Rocky Mountain Spotted Fever}

Rocky Mountain spotted fever is the most severe of the rickettsial diseases. The incidence has increased over the last decade from less than two cases per million persons in 2000 to over six million in 2010. The mortality has declined to $0.5 \%$ in the same time period. Children under 10 years, those with a compromised immune system and those with a delayed diagnosis are at an increased risk of fatality. Prior to specific antibiotic therapy, the mortality was $25 \%$ (http://www.cdc. gov/rmsf/stats/). The onset occurs 2-8 days after the bite of an infected tick, and the majority of cases occur during the summer months. High fever develops initially, followed by the pathognomonic rash, which occurs between the second and sixth days of the illness. The rash initially consists of small erythematous macules, but later these become maculopapular and petechial, and in untreated patients, confluent hemorrhagic areas may be seen. The rash first appears at the periphery and spreads up the trunk. Involvement of the palms and soles is a characteristic feature. Headache, restlessness, meningism, and confusion may occur together with other neurologic signs. Cardiac involvement with congestive heart failure and arrhythmia are common. Pulmonary involvement occurs in 10-40\% of cases. Infection is associated with an initial leukopenia, followed by neutrophil leukocytosis. Thrombocytopenia occurs in most cases [74].

Histopathologically, the predominant lesions are in the vascular system. Rickettsiae multiply in the endothelial cells, which results in focal areas of endothelial cell proliferation, perivascular mononuclear cell infiltration, thrombosis, and leakage of red cells into the tissues. The renal lesions involve both blood vessels and interstitium, and acute tubular necrosis may occur. Acute GN with 
immune-complex deposition has been reported [75], but in most cases the pathology appears to be a direct consequence of the invading organism on the renal vasculature.

Renal dysfunction is an important complication of Rocky Mountain spotted fever. Elevation of urea and creatinine levels occurs in a third of children, and acidosis is common. AKI was associated with increased mortality $-20 \%$ in a recent Mexican cohort [76]. Factors at presentation that have been associated with increased risk of developing renal impairment in adults are increasing age, rising bilirubin, thrombocytopenia, and neurological involvement. In one adult case series, factors associated with increased mortality were raised creatinine, raised bilirubin and AST, hypernatremia, thrombocytopenia, increasing age, and neurological involvement [77]. Prerenal renal failure caused by hypovolemia and impaired cardiac function may respond to volume replacement and inotropic support, but AKI may subsequently occur, necessitating dialysis.

Rocky Mountain spotted fever is diagnosed by the characteristic clinical picture, the exclusion of disorders with similar manifestations (e.g., measles, meningococcal disease, and leptospirosis), and detection of specific antibodies in convalescence. Culture of Rickettsia rickettsii, immunofluorescent staining, and polymerase chain reaction (PCR) testing of blood and skin biopsy specimens are available only in reference laboratories. Antibiotics should be administered in suspected cases without awaiting confirmation of the diagnosis [74]. Doxycycline is the drug of choice for children of any age. Chloramphenicol is also effective. Intensive support of shock and multiorgan failure may be required in severe cases, and peritoneal dialysis or hemodialysis may be required until renal function returns.

\section{Q Fever}

Q fever is caused by Coxiella burnetii and has a worldwide distribution, with the animal reservoir being cattle, sheep, and goats. Human infection follows inhalation of infected particles from the environment. The clinical manifestations range from an acute self-limited febrile illness with atypical pneumonia to involvement of specific organs that causes endocarditis, hepatitis, osteomyelitis, and central nervous system disease [78].

Proliferative GN may be associated with either Q fever endocarditis, rhabdomyolysis, or a chronic infection elsewhere in the body. Tubulointerstitial nephritis (attributed to immune-complex deposition) is usually associated with chronic rather than acute disease. The incidence of renal complications in Q fever is unknown due to the small numbers of case reports, but a recent case series of 54 patients demonstrated that $33 \%$ had renal failure [79]. Renal manifestations range from asymptomatic proteinuria and hematuria to AKI, hypertension, and nephrotic syndrome. Renal histologic findings are those of a diffuse proliferative GN, focal segmental GN, or mesangial GN. Immunofluorescent studies reveal diffuse glomerular deposits of IgM in the mesangial, together with C3 and fibrin. Coxiella burnetii antigen has not been identified within the renal lesions. IgG antibodies to cardiolipin and lupus anticoagulant have been demonstrated in acute-phase serum samples [80].

Treatment of the underlying infection may result in remission of the renal disease, but prolonged treatment may be required for endocarditis. Doxycycline is the first line recommended treatment for adults and children and has not been linked to dental staining as occurs with other tetracyclines and so can be used in children of all ages who are hospitalized with Q fever [81]. Co-trimoxazole has been used as a safe alternative (deemed safe in pregnant women) as has clarithromycin. In the acute setting, treatment should continue until 3 days after the fever has subsided (usual duration is 2-3 weeks). Chronic infection requires a longer treatment, usually 18 months (CDC recommendation). 


\section{Intravascular and Focal Bacterial Infections}

Nephritis has been reported in association with the presence of a wide range of microorganisms that cause chronic or persistent infection (Table 2). It is likely that any infectious agent that releases foreign antigens into the circulation, including those of very low virulence, can cause renal injury either by deposition of foreign antigens in the kidney or by the formation of immune complexes in the circulation, which are then deposited within the kidney. Nephritis is most commonly seen in association with intravascular infections such as SBE or infected ventriculoatrial shunts, but it is also seen after focal extravascular infections; ear, nose, and throat infections; and abscesses.

Table 2 Focal infections causing glomerulonephritis

\begin{tabular}{|c|c|}
\hline Site of infection & Organism \\
\hline \multirow[t]{13}{*}{ Infective endocarditis } & Coagulase-negative staphylococci \\
\hline & Staphylococcus aureus \\
\hline & Streptococcus pneumoniae \\
\hline & Viridans streptococci \\
\hline & Enterococci \\
\hline & Anaerobic streptococci \\
\hline & Diphtheroids \\
\hline & Haemophilus influenzae \\
\hline & Coliforms \\
\hline & Bacteroides \\
\hline & Coxiella burnetii \\
\hline & Legionella \\
\hline & Candida albicans \\
\hline \multirow[t]{5}{*}{ Shunt infections } & Coagulase-negative staphylococci \\
\hline & Staphylococcus aureus \\
\hline & Diphtheroids \\
\hline & Gram-negative bacilli \\
\hline & Anaerobes \\
\hline \multirow[t]{2}{*}{ Focal abscess } & Staphylococcus aureus \\
\hline & Gram-negative bacilli \\
\hline \multirow[t]{2}{*}{ Osteomyelitis } & Staphylococcus aureus \\
\hline & Streptococcus pyogenes \\
\hline Pyelonephritis & Coliforms \\
\hline \multirow[t]{4}{*}{ Pneumonia } & Streptococcus pneumoniae \\
\hline & Klebsiella \\
\hline & Staphylococcus aureus \\
\hline & Mycoplasma \\
\hline \multirow[t]{2}{*}{ Otitis media } & Streptococcus pneumoniae \\
\hline & Staphylococcus aureus \\
\hline \multirow[t]{4}{*}{ Gastrointestinal infection } & Yersinia \\
\hline & Campylobacter \\
\hline & Salmonella \\
\hline & Shigella \\
\hline
\end{tabular}




\section{Bacterial Endocarditis}

Renal involvement is one of the diagnostic features of bacterial endocarditis. Virtually all organisms that cause endocarditis also produce renal involvement (Table 2). Although endocarditis caused by bacteria is the most common and is readily diagnosed by blood culture, unusual but important causes of culture-negative endocarditis include Q fever and Legionella infection. In the immunocompromised individual, opportunistic pathogens such as fungi and mycobacteria are important causes.

The usual renal manifestations of SBE are asymptomatic proteinuria, hematuria, and pyuria. Loin pain, hypertension, nephrotic syndrome, and renal failure may occur in more severe cases.

The renal lesions occurring in endocarditis are variable, and focal embolic and immune-complexmediated features may coexist [82, 83]. Embolic foci may be evident as areas of infarction, intracapillary thrombosis, or hemorrhage. More commonly, there is a focal necrotizing or diffuse proliferative GN. Immunofluorescent studies show glomerular deposits of IgG, IgM, IgA, and C3 along the GBM and within the mesangium. Electron microscopy reveals typical electron-dense deposits along the GBM and within the mesangium [82].

Early reports suggested that the renal lesions were caused by microemboli from infected vegetations depositing in the kidney, a hypothesis supported by the occasional presence of bacteria within the renal lesions. Most subsequent evidence, however, indicates that immunologic mechanisms rather than emboli are involved in the pathogenesis in most cases: bacteria are rarely found within the kidney, and renal involvement occurs with lesions of the right side of the heart, which would not be likely to embolize to the kidney. Immune complexes containing bacterial antigens are present in the circulation, and both bacterial antigens and bacteria-specific antibodies can be demonstrated within the immune deposits in the kidney. Serum C3 level is usually low, and complement can be found within both the circulating and the deposited immune complexes. These features all support an immune-complex-mediated pathogenesis of the renal injury.

Treatment of the endocarditis with antibiotics usually results in resolution of the GN and is associated with the disappearance of immune complexes from the circulation and return of $\mathrm{C} 3$ levels to normal. The prognosis of the renal lesions in SBE generally depends on the response of the underlying endocarditis to antibiotics or, in cases of antibiotic failure, to surgical removal of the infective vegetations [84].

\section{Shunt Nephritis}

In patients previously treated by shunting for hydrocephalus, there is a well-documented association of GN with infected ventriculoatrial shunts. The incidence of shunt nephritis has decreased due to the preference of ventriculoperitoneal shunts over ventriculoatrial shunts; however, shunt nephritis has been reported with the former [85]. Coagulase-negative staphylococci are the causative organisms in $75 \%$ of cases. It is an immune-complex disease with chronic hyperantigenemia, hyperglobulinemia, and the deposition of immunoglobulins, complement, and immune complexes on the glomerular basement membrane [86]. The clinical and pathologic findings are similar to those in SBE. Presenting features are proteinuria, hematuria, and pyuria, and they may progress to renal failure. Immune complexes containing the bacterial antigens and complement are present in the serum, and C3 is depressed. Histologic findings are those of a diffuse mesangiocapillary GN. The prognosis for the renal lesion is good if the infection is treated early. This usually involves removal of the infected shunt or replacement of a VA with a VP shunt and administration of appropriate antibiotics, though possible progression to end-stage renal disease requires frequent renal monitoring of patients with ventriculoatrial shunts [87]. Certain case reports have also seen benefit with the use of steroids as adjunctive therapy [88]. 


\section{Other Focal Infections}

GN has been reported after chronic abscesses, osteomyelitis, otitis media, pneumonia, and other focal infections (Table 2). AKI has been the presenting feature of focal infections in various sites, including the lung, pleura, abdominal cavity, sinuses, and pelvis. Many different organisms have been responsible, including S. aureus, Pseudomonas, E. coli, and Proteus species. This is probably another example of immune-complex GN. C3 level is decreased in approximately one-third of reported cases, and immunofluorescent studies reveal diffuse granular deposits of $\mathrm{C} 3$ in the glomeruli of all reported instances, with a variable presence of immunoglobulin. The renal lesion is that of MPGN and crescentic nephritis. The renal outcome is reported to be good with successful early treatment of the underlying infection.

\section{Viral Infections}

The role of viral infections in the causation of renal disease has been less well defined than that of bacterial infections. Clearly defined associations of renal disease have been made with hepatitis B virus (HBV), hepatitis $\mathrm{C}$ virus (HCV), HIV, and hantaviruses, but the role of most other viruses in the pathogenesis of renal disease is not clearly defined. Most viruses causing systemic infection may trigger immunologically mediated renal injury. With increasing application of molecular techniques, it may be that a significant proportion of GNs currently considered to be idiopathic will ultimately be shown to be virus induced. In children with immunodeficiency states and those undergoing renal transplantation, viruses such as cytomegalovirus (CMV) and polyomavirus have been recognized to be associated with nephropathy.

\section{Hepatitis B Virus}

About 2,000 million people alive today have been infected with hepatitis B at some point in their lives, of these 350 million become chronically infected and are carriers of the disease. There are an estimated four million acute cases per year, and per year $25 \%$ of carriers (one million) die from cirrhosis, chronic active hepatitis, or primary hepatocellular carcinoma. The infection is most common in Africa and the Orient, where it is acquired in childhood by vertical transmission from infected mothers or by horizontal transmission from other children or adults. In developed countries, transmission in adults occurs more often by blood product exposure, sexual contact, or intravenous drug use. The epidemiology of HBV infection in children is changing following the widespread use of effective vaccination at birth, in both developed and developing countries.

HBV is a complex DNA virus with an outer surface envelope (HBsAg) and an inner nucleocapsid core containing the hepatitis B core antigen ( $\mathrm{HBcAg})$, DNA polymerase, protein kinase activity, and viral DNA. Incomplete spherical and filamentous viral particles consisting solely of HBsAg are the major viral products in the circulation and may be present in concentrations of up to 1,014 particles per milliliter of serum. Hepatitis B e antigen ( $\mathrm{HBeAg}$ ) can be released from $\mathrm{HBcAg}$ by proteolytic treatment and may be found in the circulation either free or complexed to albumin or IgG antibodies. The presence of HBeAg correlates with the presence of complete viral particles and the infectivity of the individual. Infection with HBV may result in either a self-limited infectious hepatitis followed by clearance of the virus and complete recovery, or a chronic or persistent infection in which the immune response is ineffective in eliminating the virus. Chronic HBV infection with continued presence of viral antigens in the circulation caused by an ineffective host immune response provides the best-documented example of immunologically mediated renal injury caused by persistent infection. The risk of chronic infection varies inversely with age with $90 \%$ of infants infected at birth developing chronic infection, compared 
to $25-50 \%$ of children infected between 1 and 5 years and $1-5 \%$ of people infected as older children and adults. Chronic infection is also common in those with immunodeficiency.

\section{Patterns of Hepatitis B Virus Immunologically Mediated Renal Disease}

Several forms of renal disease secondary to hepatitis B infection have been identified including membranous glomerulonephritis, mesangioproliferative glomerulonephritis, as well as IgA nephropathy and focal segmental glomerulosclerosis (FSGS) [89]. Hepatitis B has also been linked with polyarteritis nodosa (PAN).

Prodromal Illness. In the early prodromal phase of HBV hepatitis, before the onset of jaundice, some patients develop fever, maculopapular or urticarial rash, and transient arthralgias or arthritis. Occasionally, proteinuria, hematuria, or sterile pyuria is observed. The syndrome usually lasts 3-10 days and often resolves before the onset of jaundice [90]. There have been no histologic studies of the renal changes during this prodromal period.

Hepatitis B Virus-Associated Polyarteritis Nodosa. HBV infection is associated with polyarteritis nodosa, a vasculitis affecting the small- and medium-sized vessels (HBV PAN). Most of these cases have been in adults, but the disorder has also been reported in children, where it is estimated that approximately one-third of PAN cases are caused by HBV [91, 92]. HBV PAN appears to be uncommon in Africa and the Orient, where infection is usually acquired in childhood, and has declined in incidence following the introduction of HBV vaccination [93].

HBV PAN presents weeks to months after a clinically mild hepatitis but may occasionally predate the hepatitis. After a prodromal illness, frank vasculitis affecting virtually any organ appears. Abdominal pain, fever, mononeuritis multiplex, and pulmonary and renal involvement may occur and can be the first clinical presentation of hepatitis B [94]. The renal involvement may appear as hypertension, hematuria, proteinuria, or renal failure. Laboratory investigations reveal a florid acutephase response, leukocytosis, and anemia. Transaminase levels are usually elevated, and HBsAg is present in the circulation. The pathology consists of focal inflammation of small- and medium-sized arteries, with fibrinoid necrosis, leukocyte infiltration, and fibrin deposition. Renal pathology may be limited to the medium-sized arteries or may coexist with GN [95]. Circulating immune complexes containing HBsAg and anti-HBs antibodies are usually present in the circulation. $\mathrm{C} 3, \mathrm{C} 4$, and total hemolytic complement levels are depressed. A positive ANCA excludes HBV PAN [93]. Although most evidence suggests that the pathogenesis involves an immune-complex-mediated vasculitis, autoantibody- or cell-mediated vascular injury may coexist. If the condition is untreated, the mortality is high. Most studies suggest that steroids or immunosuppressants help to suppress the vasculitis but potentially predispose to chronic infection or progressive liver disease. Successful treatment of hepatitis B-associated PAN with nucleoside analogues such as lamivudine or newer antiviral drugs, either alone or in combination with interferon-alpha and conventional immunosuppressive therapy, has been reported [93, 96].

Hepatitis B Virus-Associated Membranous Glomerulonephritis. HBV is now the major cause of membranous GN (MGN) in children worldwide. The proportion of patients with MGN caused by $\mathrm{HBV}$ is directly related to the incidence of HBsAg in the population, with $80-100 \%$ of all cases of MGN in some African and Oriental countries being associated with HBV (see chapter " Membranous Nephropathy"). HBV MGN usually presents in children aged 2-12. There is a striking male predominance. The virus is usually acquired by vertical transmission from infected mothers or horizontally from infected family members. Unlike adults with HBV MGN, children do not usually have a history of hepatitis or of active liver disease, but liver function test results are generally mildly abnormal. Liver biopsy specimens may show minimal abnormalities, chronic persistent hepatitis, or (occasionally) more severe changes. 
The renal manifestations are usually of proteinuria, nephrotic syndrome, microscopic hematuria, or (rarely) macroscopic hematuria. Hypertension occurs in less than $25 \%$ of cases, and renal insufficiency is rare. HBsAg and $\mathrm{HBcAg}$ are usually present in the circulation, and $\mathrm{HBe}$ antigenemia is seen in a high proportion of cases. Occasionally, HBsAg may be found in the glomeruli but is absent from the circulation. $\mathrm{C} 3$ and $\mathrm{C} 4$ levels are often low, and circulating immune complexes are found in most cases. Immunohistologic study reveals deposits of IgG and C3 and (less commonly) IgM and IgA in subepithelial, subendothelial, or mesangial tissue. HBV particles may be seen on electron microscopy, and all the major hepatitis B antigens, including $\mathrm{HBsAg}, \mathrm{HBcAg}$, and $\mathrm{HBe} \mathrm{Ag}$, have been localized in the glomerular capillary wall on immunofluorescence.

Immunologic deposition of HBV and antibody in the glomerular capillary wall is clearly involved in the glomerular injury, but the underlying immunologic events are incompletely understood [90]. Passive trapping of circulating immune complexes may be involved, but the circulating immune complexes containing HBsAg are usually larger than would be expected to penetrate the basement membrane. $\mathrm{HBsAg}$ and $\mathrm{HBcAg}$ are anionic and are therefore unlikely to penetrate the glomerular capillary wall. In contrast, $\mathrm{HBeAg}$ forms smaller complexes with anti-HBe antibodies and may readily penetrate the GBM. This may explain the observation that HBeAg in the circulation frequently correlates with the severity of the disease [90]. An alternative mechanism for immunemediated glomerular injury is the trapping of HBV antigens by antibody previously deposited in the kidney. Anti-HBe antibodies are cationic and may readily localize in the glomerulus and subsequently bind circulating antigen and complement. The third possibility is that the depositions of HBV and antibodies are consequences of glomerular injury by cellular mechanisms or autoantibodies. Little evidence supports this view at present [90]. A transgenic mouse model of $\mathrm{HBV}$-associated nephropathy has been developed, in which HBsAg and HBcAg is expressed in the liver and kidney, particularly tubular epithelial cells, without viral replication. In these mice, gene expression analysis revealed upregulation of acute-phase proteins, particularly $\mathrm{C} 3$, although measurable serum C3 levels were reduced. This supports the notion that local persistent expression of HBV viral proteins contributes to HBV-associated nephropathy [97].

Other Hepatitis B Virus Glomerulonephritides. HBV infection has been associated with a variety of other forms of GN in both adults and children [95]. Both MPGN and mesangial proliferative GN may be triggered by HBV. In several countries where HBV is common, the proportion of patients with these forms of nephritides who test positive for HBV greatly exceeds the incidence of positivity in the general population. As with MGN and HBV-associated PAN, circulating immune complexes and localization of HBV antigens in the glomeruli have been reported in both MPGN and mesangial proliferative GN, and it is likely that similar mechanisms are occurring [90]. Several other forms of GN have been associated with HBV, including IgA nephropathy, focal glomerulosclerosis, crescentic nephritis, and systemic lupus erythematosus, but the evidence for these associations is less consistent than for the entities discussed earlier [95].

\section{Treatment of Hepatitis B Virus Glomerulonephritis}

$\mathrm{HBV}$ is normally cleared as a result of cell-mediated responses in which cytotoxic T cells and natural killer cells eliminate infected hepatocytes. It is not surprising, therefore, that the administration of steroids and immunosuppressive agents either may have no effect on HBV disease or may increase the risk of progressive disease. Children with HBV MGN have a good prognosis, and two-thirds undergo spontaneous remission within 3 years of diagnosis. Steroid therapy does not appear to provide any additional benefit $[90,98]$. Antiviral therapy with pegylated interferon-alpha and lamivudine is used to treat $\mathrm{HBV}$, and in some cases, elimination of the infection with antiviral therapy in both children and adults is associated with improvement or resolution of the coexisting renal disease [99]. There are 
isolated case reports of pegylated interferon successfully treating IgA nephropathy associated with HBV [100]. There have been some promising results with newer antiviral agents such as adefovir and entecavir in treating HBV glomerulonephritis in combination regimens [101].

\section{Hepatitis C Virus}

$\mathrm{HCV}$ is an enveloped, single-stranded RNA virus of approximately 9.4 kilobases in the Flaviviridae family. There are six major HCV genotypes. Hepatitis $\mathrm{C}$ is a common disease affecting approximately 400 million people worldwide. In the United States, 4.1 million persons are estimated to be anti-HCV positive, and 3.2 million may be chronically infected [102]. An estimated 240,000 children in the United States have antibody to HCV, and 68,000-100,000 are chronically infected. Children become infected through receipt of contaminated blood products or through vertical transmission. The risk of vertical transmission increases with higher maternal viremia and maternal coinfection with HIV.

Acute HCV infection is rarely recognized in children outside of special circumstances such as a known exposure from an $\mathrm{HCV}$-infected mother or after blood transfusion. Most chronically infected children are asymptomatic and have normal or only mildly abnormal alanine aminotransferase levels. Although the natural history of HCV infection during childhood seems benign in the majority of instances, the infection can take an aggressive course in a proportion of children, leading to cirrhosis and end-stage liver disease during childhood. The factors responsible for this more aggressive course are unidentified [103]. Even in adults, the natural history of HCV infection has a variable course, but a significant proportion of patients will develop some degree of liver dysfunction, and 20-30\% will eventually have end-stage liver disease as a result of cirrhosis. The risk of hepatocellular carcinoma is significant for those who have established cirrhosis. Hepatitis $\mathrm{C}$ is currently the most common condition leading to liver transplantation in adults in the "Western world."

GN has been described as an important complication of chronic infection with HCV in adults [104]. The clinical presentation is usually of nephrotic syndrome or proteinuria, hypertension, or hematuria, with or without azotemia. MPGN, with or without cryoglobulinemia, and MGN are most commonly described [105]. Isolated case reports of other, more unusual patterns of glomerular injury, including IgA nephropathy, focal segmental glomerulosclerosis, crescentic GN, fibrillary GN, and thrombotic microangiopathy, have also been associated with HCV infection [106]. Glomerular deposition of hepatitis antigens and antibodies has been described and is believed to play a role in pathogenesis. Cryoglobulinemia is a common accompaniment of GN that is associated with the depression of serum complement levels [106]. Renal failure may develop in $40-100 \%$ of patients who have MPGN. The presence of viruslike particles as well as viral RNA within the kidney sections of patients with $\mathrm{HCV}$-associated glomerulopathies has been reported [107]. The diagnosis should be suspected if glomerular disease is associated with chronic hepatitis, particularly with the presence of cryoglobulins, but renal biopsy is necessary to establish a definitive diagnosis.

$\mathrm{HCV}$ infection is relatively common in children with end-stage renal disease and is an important cause of liver disease in this population. HCV-infected renal transplant recipients had higher mortality and hospitalization rates than other transplant recipients [108], and HCV infection has been reported to be associated with de novo immune-mediated GN, especially type 1 MPGN, in renal allografts, resulting in accelerated loss of graft function. The PEDS-C trials, a randomized controlled trial in children and adolescents, have shown that ribavirin and pegylated interferon is superior to pegylated interferon and placebo in treating chronic hepatitis $\mathrm{C}$ in this age group, and this combination constitutes the US standard of care [109]. Clinical trials are currently underway investigating the efficacy of a new generation of HCV antiviral drugs, including sofosbuvir, 
boceprevir, and telaprevir in combination with pegylated interferon and ribavirin. Triple therapy has been associated with a higher sustained virological response in adults [110].

Hepatitis $\mathrm{C}$ may be complicated by systemic mixed cryoglobulinemic (MC) vasculitis and in some cases by a polyarteritis nodosa (PAN)-type non-cryoglobulinemic vasculitis [111]. Treatment with interferon- $\alpha$ (IFN- $\alpha)$ and ribavirin mostly is associated with an improvement of vasculitic symptoms. In some cases, exacerbation and rarely new onset of vasculitis of the peripheral nervous system have been described after this treatment. In fulminant cases, immunosuppressive therapy with steroids, and cyclophosphamide, or rituximab may be needed to control life-threatening vasculitis prior to antiviral treatment [111].

\section{Herpes Viruses: Cytomegalovirus}

CMV is one of the eight human herpes viruses. Transmission of the virus requires exposure to infected body fluids such as breast milk, saliva, urine, or blood. Individuals initially infected with CMV may be asymptomatic or display nonspecific flulike symptoms. After the initial infection CMV, like all herpes viruses, establishes latency for life but will be periodically excreted by an asymptomatic host. CMV replicates within renal cells, and on biopsy samples from immunocompromised hosts, viral inclusions can be visualized by light microscopy in cells of the convoluted tubules and collecting ducts [112]. Glomerular cells and shed renal tubular cells may have characteristic inclusions, but clinically evident renal disease is rare and is seen virtually only in immunocompromised or congenitally infected children.

The clinical manifestations of CMV-induced renal disease in congenitally infected infants are variable and range from asymptomatic proteinuria to nephrotic syndrome and renal impairment. In congenital CMV infection, histologic changes of viral inclusions commonly occur in the tubules. In addition, proliferative $\mathrm{GN}$ has been reported, with evidence on electron microscopy of viral immune deposits in glomerular cells [113]. In CMV-infected immunocompromised patients, immunecomplex GN has been documented with mesangial deposits of $\operatorname{IgG}, \operatorname{IgA}, \mathrm{C} 3$, and CMV antigens within glomeruli. Eluted glomerular immunoglobulins have been shown to contain CMV antigens [114].

$\mathrm{CMV}$ is the most common viral infection after kidney transplantation. Experience with pediatric kidney transplant recipients suggests a $67 \%$ incidence of CMV infection, with approximately $67 \%$ being negative at the time of transplantation [115]. The direct and indirect effects of CMV infection result in a significant morbidity and mortality among kidney transplant recipients. CMV-negative patients who receive a CMV-positive allograft are at risk for primary infection and graft dysfunction. Patients who are CMV seropositive at the time of transplantation are also at risk of reactivation and superinfection. Tubulointerstitial nephritis is a well-characterized pathologic feature of renal allograft CMV disease, which can be difficult to distinguish from injury caused by rejection. Histologic evidence of endothelial cell injury and mononuclear cell infiltration in the glomeruli has been reported. CMV glomerular vasculopathy in the absence of tubulointerstitial disease, causing renal allograft dysfunction, has also been reported [116]. Beyond the acute allograft nephropathy associated with CMV viremia, CMV is known to cause chronic vascular injury. This may adversely affect the long-term outcome of the allograft and may be the explanation for the observed association with chronic allograft nephropathy.

Techniques for rapidly diagnosing CMV infection include shell vial culture, pp65 antigenemia assay, PCR, and the hybrid-capture RNA-DNA hybridization assay for qualitative detection of CMV PCR. Reverse transcriptase PCR (RT PCR) can detect viral mRNA transcripts in peripheral blood but is less sensitive than pp65 or PCR [117]. Immune histocytochemistry is used for diagnosing end-organ disease. Quantitative plasma PCR testing (PCR viral load) is increasingly 
used for diagnosis and monitoring of CMV viremia in renal transplant recipients [117]. The American Society of Transplantation (AST) recommends monitoring for CMV by quantitative viral load monthly for the first year after transplantation, and the Kidney Disease Improving Global Outcomes (KDIGO) recommends an additional 3 monthly surveillance for the second year.

Antiviral agents that have been shown to be effective against CMV include ganciclovir, valganciclovir, foscarnet, and cidofovir. Ganciclovir remains the drug of choice for treating established disease. Intravenous ganciclovir therapy is preferred in children because of the erratic absorption of oral ganciclovir. Major limitations of ganciclovir therapy are the induction of renal tubular dysfunction and bone marrow toxicity, principally neutropenia and thrombocytopenia. Dosage adjustments are necessary for recipients with renal dysfunction. Oral valganciclovir is recommended for CMV prophylaxis posttransplant [118]. Duration of chemoprophylaxis is dependent on the serostatus of the donor and recipient [115]. While it is effective in reducing the incidence of symptomatic CMV, prospective viral surveillance studies have shown subclinical infection in $12-22 \%$ of pediatric kidney transplant recipients, hence the importance of viral surveillance [115]. Use of other antiviral agents such as foscarnet and cidofovir is limited because of nephrotoxicity and difficulty of administration. The role of high-titer CMV immunoglobulin therapy in reducing severe CMV-associated disease after stem cell transplant remains unclear [119].

\section{Herpes Viruses Varicella-Zoster Virus}

The association of varicella with nephritis has been known for more than 100 years since Henoch reported on four children with nephritis that occurred after the appearance of varicella vesicles. Varicella, however, is rarely associated with renal complications. In fatal cases with disseminated varicella and in the immunocompromised individual, renal involvement is more common. Histologic findings in fatal cases include congested hemorrhagic glomeruli, endothelial cell hyperplasia, and tubular necrosis. In mild and nonfatal cases and in nonimmunocompromised individuals, varicella is occasionally associated with a variety of renal manifestations, ranging from mild nephritis to nephrotic syndrome and AKI [120]. Histologic findings include endocapillary cell proliferation, epithelial and endothelial cell hyperplasia, and inflammatory cell infiltration. Rapidly progressive nephritis has also been reported. Immunohistochemical studies reveal glomerular deposition of IgG, IgM, IgA, and C3. On electron microscopy, granular electron-dense deposits have been found in the paramesangial region, and varicella antigens may be deposited in the glomeruli. The features suggest an immune-complex nephritis. Elevated circulating levels of IgG and IgA immune complexes and depressed C3 and C4 levels support this possibility [121].

Fulminant disseminated varicella and varicella in immunocompromised patients should be treated with intravenous acyclovir.

\section{Herpes Viruses: Epstein-Barr Virus}

Renal involvement is common during acute infectious mononucleosis, usually manifesting as an abnormal urine sediment, with hematuria in up to $60 \%$ of cases. Hematuria, either microscopic or macroscopic, usually appears within the first week of the illness and lasts for a few weeks to a few months. Proteinuria is usually absent or low grade. More severe renal involvement with proteinuria, nephrotic syndrome, or acute nephritis with renal failure is much less common. AKI may be seen during the course of fulminant infectious mononucleosis with associated hepatic failure, thrombocytopenia, and encephalitis. It is usually caused by interstitial nephritis that is likely the result of immunopathologic injury precipitated by Epstein-Barr virus (EBV) infection. However, the identification of EBV DNA in the kidney raises the possibility that direct infection might play a role [122, 123]. The renal involvement must be distinguished from myoglobinuria caused by rhabdomyolysis, 
which may occur in infectious mononucleosis, and from bleeding into the renal tract as a result of thrombocytopenia.

Renal histologic findings in EBV nephritis are an interstitial nephritis with mononuclear cell infiltration and foci of tubular necrosis. Glomeruli may show varying degrees of mesangial proliferation. On immunohistochemical study, EBV antigens are seen in glomerular and tubular deposits. The prognosis for complete recovery of renal function is good. Treatment with corticosteroids may have a role in the management of EBV-induced AKI and may shorten the duration of renal failure [124]. EBV-associated posttransplantation lymphoproliferative disease is a recognized complication in renal transplant recipients. Subclinical infection occurs in $35-40 \%$ of pediatric renal transplant recipients [115]. Latent infection of EBV in renal proximal tubular epithelial cells has been described as causing idiopathic chronic tubulointerstitial nephritis [125].

\section{Herpes Viruses: Herpes Simplex Virus}

The herpes simplex virus (HSV) causes persistent infection characterized by asymptomatic latent periods interspersed with acute relapses. As with other chronic and persistent infections, immunologically mediated disorders triggered by HSV are well recognized, and it is perhaps surprising that HSV has rarely been linked to nephritis. Acute nephritis and nephrotic syndrome have been associated with herpes simplex encephalitis. Renal histology shows focal segmental GN with mesangial and segmental deposits of IgM, C3, and HSV antigens. As with other herpes viruses, HSV has been suggested as a trigger for IgA nephritis, MPGN, and membranous nephropathy, but no conclusive evidence exists of an etiologic role for HSV.

\section{Human Immunodeficiency Virus}

The WHO estimate that in 2012 there were 35.3 million people living with HIV worldwide, with 3.3 million being children. Of these 260,000 were new infections (www.unaids.org - UNAIDS Global Report on the global AIDS epidemic 2013). The number of children under 15 years of age receiving antiretroviral therapy (ART) in low- and middle-income countries rose from 566,000 in 2011 to 630,000 in 2012 (www.unaids.org - Global update on HIV treatment 2013).

Renal involvement occurs in 2-15\% of HIV-infected children in the United States [126-128]. Since the development of antiretroviral therapy (ART), however, the incidence of end-stage renal disease in HIV infection in both adults and children in industrialized countries has declined, but it is predicted that the dramatic decline in AIDS-related deaths will lead to an ageing population of HIV-infected individuals who will be at risk of non-HIV-related renal problems, such that the numbers of HIV-positive ESRD patients will increase in the United States [129].

HIV infection is associated with a number of renal pathologies. HIV-associated nephropathy (HIVAN) is a syndrome of glomerular and tubular dysfunction, which can progress to end-stage renal failure. It is discussed more fully below. Glomerular syndromes other than HIVAN include a range of immune-mediated syndromes (HIV immune-complex kidney disease (HIVICK)) including MGN that resembles lupus nephritis and immune-complex GN, with IgA nephropathy and HCV-associated MPGN being the most common forms [126, 127]. There have also been several case reports of amyloid kidney. The kidneys may be affected by various other mechanisms. Opportunistic infections with organisms such as BK virus (BKV) that give rise to nephropathy and hemorrhagic cystitis have been reported in association with HIV infection [130]. Systemic infections accompanied by hypotension can cause prerenal failure leading to acute tubular necrosis. Acute tubular necrosis has also been reported in HIV patients after the use of nephrotoxic drugs such as pentamidine, foscarnet, cidofovir, amphotericin B, and aminoglycosides. Intratubular obstruction with crystal precipitation can occur with the use of sulfonamides and intravenous acyclovir. Indinavir is well recognized to cause 
nephropathy and renal calculi [131]. MPGN associated with mixed cryoglobulinemia and thrombotic microangiopathy/atypical HUS in association with HIV infections have been reported [132, 133].

\section{HIV-Associated Nephropathy (HIVAN)}

HIVAN is characterized by both glomerular and tubular dysfunction, the pathogenesis of which is not entirely known. HIVAN is a clinicopathologic entity that includes proteinuria, azotemia, focal segmental glomerulosclerosis or mesangial hyperplasia, and tubulointerstitial disease [128]. In adults in the United States, there is a markedly increased risk of nephropathy among African American persons with HIV infection. This appears to be true in children as well, but the data are sparse. The spectrum of HIVAN seems to be coincident with the degree of AIDS symptomatology. It is thought that HIVAN can present at any point in HIV infection, but most patients with HIVAN have CD4 counts of less than $200 \times 10^{6}$ cells $/ \mathrm{mL}$, which suggests that it may be primarily a manifestation of late-stage disease [134].

The microscopic features of HIVAN in children comprise of classical FSGS with or without mesangial hyperplasia combined with microcystic tubular dilatation and interstitial inflammation [135]. Collapsing FSGS is the hallmark of adult disease, and this has been shown in children as well. In the affected glomeruli, visceral epithelial cells are hypertrophied and hyperplastic and contain large cytoplasmic vacuoles and numerous protein resorption droplets. There is microcystic distortion of tubule segments, which contributes to increasing kidney size. Podocyte hyperplasia can become so marked that it causes obliteration of much of the urinary space, forming "pseudocrescents" [136]. Capillary walls are wrinkled and collapsed with obliteration of the capillary lumina. The interstitium is edematous with a variable degree of T-cell infiltration. The Bowman capsule can also be dilated and filled with a precipitate of plasma protein that represents the glomerular ultrafiltrate. One of the most distinctive features of HIVAN, however, is the presence of numerous tubuloreticular inclusions within the cytoplasm of glomerular and peritubular capillary endothelial cells [136]. Immunofluorescence testing is positive for IgM and C3 in capillary walls in a coarsely granular to amorphous pattern in a segmental distribution [137].

The presence of the HIV genome in glomerular and tubular epithelium has been demonstrated using complementary DNA probes and in situ hybridization. Proviral DNA has been detected by PCR in the glomeruli, tubules, and interstitium of microdissected kidneys from patients who had pathologic evidence of HIVAN, but it has also been detected in the kidneys of HIV-positive patients with other glomerulopathies and in those with undetectable levels of viral RNA in the peripheral blood [138]. The kidney has been postulated to behave as a separate compartment to the blood with ongoing viral replication in the kidneys despite achieving serological suppression with ART $[138,139]$.

Transgenic murine models provide some of the strongest evidence for a direct role of HIV-1 in the induction of HIVAN. These mice do not produce infectious virus but express the HIV envelope and regulatory genes at levels sufficient to recreate the HIVAN that is seen in humans. Animal models have shown that the nef and $v p r$ genes contribute to HIVAN through encoding for podocyte dysfunction and apoptosis of renal tubular epithelial cells, respectively [135]. HIV is thought to infect renal tubuloepithelial cells through direct cell-cell transmission which then act as a separate viral compartment and facilitates replication distant from the blood. HIVAN 1 and 2 are the host susceptibility genes identified in animal models for HIVAN. Two variants (g1and G2) in the ApoL1 gene have been identified as the susceptibility alleles that contribute to the increased risk of FSGS in African Americans (previously attributed to MYH9 on chromosome 22) [135]. HIVAN is more likely in patients with a family history of ESRD.

HIVAN can manifest as mild proteinuria, nephrotic syndrome, renal tubular acidosis, hematuria, and/or AKI [128]. Nephrotic syndrome and chronic renal insufficiency are late manifestations of HIVAN. Children with HIVAN are likely to develop transient electrolytic disorders, heavy proteinuria, 
and AKI due to systemic infectious episodes or nephrotoxic drugs. Early stages of HIVAN can be identified by the presence of proteinuria and "urine microcysts" along with renal sonograms showing enlarged echogenic kidneys. Urinary renal tubular epithelial cells are frequently grouped together to form these microcysts, which were found in the urine of children with HIVAN who had renal tubular injury [128]. Advanced stages of HIVAN typically present with nephrotic syndrome with edema, heavy proteinuria, hypoalbuminemia, and few red or white blood cells in urinary sediments. Hypertension may be present, but usually blood pressure is within or below the normal range. HIVAN in adults follows a rapidly progressive course, with end-stage renal disease developing within 1-4 months, but in children this rapid progression does not necessarily occur. Definitive diagnosis of HIVAN should be based on biopsy results, and biopsy should be performed if a significant proteinuria is present, because in approximately $50 \%$ of HIV-infected patients with azotemia and/or proteinuria $(>1 \mathrm{~g} / 24 \mathrm{~h})$ who undergo renal biopsy, the specimen will have histologic features consistent with other renal diseases [134].

\section{HIV Immune-Complex Kidney Disease (HIVICK)}

HIVCK is thought to arise by deposition of immune complexes or by in situ formation of immune complexes in the parenchyma [126]. The immune complexes consist of HIV antigens bound to IgG and IgA. IgA nephropathy, membranous and membranoproliferative glomerulonephritis, and a lupus-like glomerulonephritis are included in HIVCK. Like HIVAN, it occurs in Afro-Caribbeans but can also be found in Caucasians [126].

Acute interstitial nephritis (AIN) results mainly from medications used to treat HIV and its complications. Nonsteroidal anti-inflammatory drugs, rifampicin, co-trimoxazole, and protease inhibitors (PI) like idinavir and ritonavir have been implicated [140]. All classes of ART can cause renal toxicity except the integrase inhibitors and the CCR5 antagonists. Tenofovir can cause proximal tubular nephropathy and can present as complete or partial Fanconi syndrome [141, 142]. Therefore, it is essential that children on ART have regular urinalysis to check for the emergence of proteinuria and hematuria.

HAART should be given to children with symptomatic HIV disease. Specific treatment of HIVAN remains controversial. Several studies have looked at the role of HAART with or without angiotensin I-converting enzyme (ACE) inhibitors, and even cyclosporin with somewhat encouraging results. However, as yet, no randomized case-controlled trials have been undertaken [126]. Most of the studies have been small and retrospective, and many have included patients both with and without renal biopsy-proven HIVAN. Cyclosporin has been used to treat HIVAN in children with remission of nephrotic syndrome [143]. Similar responses have been reported to treatment with corticosteroids in various studies; however, steroids are not currently recommended for the routine management of HIVAN. There has been short-term improvement in kidney function in children with lymphoid interstitial pneumonitis, but there is the risk of exacerbation of infection with TB when used in developing countries, and in the absence of HAART, it has not been shown to limit the progression of HIVAN in children [126-128].

The general regimen used to treat patients with HIV, including HAART, should be applied to children with HIVAN. The dosages of some medications must be adjusted to the patients' glomerular filtration. There are reports of spontaneous regression of HIVAN with supportive management and treatment with HAART, particularly with regimens containing protease inhibitors [144]. The kidneys of transgenic mice have been found to have elevated levels of TGF- $\beta$ messenger RNA and protein [145]. Furthermore, gene expression analysis on tubular epithelial cells from a patient with HIVAN found upregulation of several inflammatory mediator genes downstream of interleukin 6 and of the transcription factor NFkB [146]. Several therapeutic options have been aimed specifically at the presumed role of TGF- $\beta$ in the pathogenesis of HIVAN. Treatment directed at its 
synthesis using gene therapy to block TGF- $\beta$ gene expression is being explored. Therapy directed at decreasing the activity of TGF- $\beta$ using anti-TGF- $\beta$ antibodies or other inhibitory substances is also an area of investigation. To date, these novel therapeutic approaches have not yielded any promising advancement in treatment.

In the HAART era, the outlook for HIV patients with ESRD has improved, but these patients fare worse than ESRD patients without HIV [147]. Most reports of HIV-infected patients on hemodialysis have shown poor prognosis, with mean patient survival times ranging from 14 to 47 months. Mortality is therefore still close to $50 \%$ within the first year of dialysis. In general, improved survival is associated with younger age at initiation of hemodialysis and with higher CD4 counts. Complications such as infection and thrombosis tend to occur at a higher rate in HIV-infected hemodialysis patients. Cross infection with HIV in dialysis patients is very rare. Peritoneal dialysis is an alternative for HIV-infected patients. The incidence of peritonitis varies across studies, but some studies did report a higher incidence of Pseudomonas and fungal peritonitis in the HIV-positive population [148]. Infections with unusual organisms such as Pasteurella multocida, Trichosporon beigelii, and Mycobacterium avium-intracellulare complex have also been reported. Several studies, however, have suggested that there is no significant difference between the HIV-infected and non-HIV-infected populations. Of note is that virus capable of replication in vitro has been recovered from the peritoneal dialysis effluent, and it can be recoverable for up to 7 days in dialysis bags at room temperature and for up to $48 \mathrm{~h}$ in dry exchange tubing [148].

Kidney transplantation in HIV-infected individuals with end-stage renal disease has shown excellent 3-5-year survival rates [149, 150]. These group of patients do experience an increase risk of rejection but not of opportunistic infections. Most issues revolve around immunosuppressive therapy and interactions with ART.

\section{Human Polyomaviruses}

The human polyomaviruses are members of the papovavirus family and significant pathogens in immunocompromised patients. They are non-enveloped viruses ranging in size from 45 to $55 \mathrm{~nm}$, with a circular, double-stranded DNA genome that replicates in the host nucleus. The best-known species in this genus are the BKV, the JC virus (JCV), and the simian virus SV40. BKV establishes infection in the kidney and the urinary tract, and its activation causes a number of disorders, including nephropathy and hemorrhagic cystitis. BKV-associated nephropathy is a cause of renal dysfunction in renal transplantation patients [151]. JCV establishes latency mainly in the kidney, and its reactivation can result in the development of progressive multifocal leukoencephalopathy. There are a few reports of nephropathy in association with JCV infection [152, 153], but BKV poses a much bigger problem in this regard.

\section{BK Virus}

BKV infection is endemic worldwide. Seroprevalence rates as high as $60-80 \%$ have been reported among adults in the United States and Europe. The peak incidence of primary infection (as measured by acquisition of antibody) occurs in children 2-5 years of age. BKV antibody may be detected in as many as $50 \%$ of children by 3 years of age, and in $60-100 \%$ of children by 9 or 10 years of age; antibodies wane thereafter. BKV infection may be particularly important in the pediatric transplantation population, in whom primary infection has a high probability of occurring while the children are immunosuppressed [151].

Primary infection with BKV in healthy children is rarely associated with clinical manifestations. Mild pyrexia, malaise, vomiting, respiratory illness, pericarditis, and transient hepatic dysfunction have been reported with primary infection. Investigators hypothesize that after an initial round of 
viral replication at the site of entry, viremia follows with dissemination of the virus to distant sites at which latent infection is established. The most frequently recognized secondary sites of latent infection are renal and uroepithelial cells. Reactivation and urinary shedding occurs in 10-60\% of immunocompetent individuals, with higher rates among the immunocompromised [154]. Secondary infection has been reported to cause tubulointerstitial nephritis and ureteral stenosis in renal transplantation patients. It may be that renal impairment in immunocompromised patients and in nonrenal solid organ transplant recipients is found to be frequently associated with BKV infection.

\section{BK Virus Nephropathy in Patients Undergoing Renal Transplantation}

The reported prevalence of BKV nephropathy in renal allografts is between $1 \%$ and $8 \%$ [155]. Asymptomatic infection is characterized by viral shedding without any apparent clinical features. Viruria, resulting from either primary or secondary infection, can persist from several weeks to years. Tubulointerstitial nephritis associated with BKV in renal transplant recipients is accompanied by histopathologic changes, with or without functional impairment. "Infection" and "disease" must be differentiated carefully. BKV infection (either primary or reactivated) can progress to BKV disease, but will not always do so [156]. Furthermore, not all cases of BKV disease lead to renal impairment. However, infection can progress to transplant dysfunction and graft loss, although the diagnosis may be complicated by the coexistence of active allograft rejection.

BKV nephritis has a bimodal distribution, with $50 \%$ of BKV-related interstitial nephritis cases occurring 4-8 weeks after transplantation and the remainder of patients developing disease months to years after transplantation. Allograft failure is due mainly to extensive viral replication in tubular epithelial cells leading to frank tubular necrosis [153]. Although damage is potentially fully reversible early in the disease, persisting viral damage leads to irreversible interstitial fibrosis. Tubular atrophy and allograft loss has been observed in $45 \%$ of affected patients. In most cases, BKV nephropathy in adult renal transplant recipients represents a secondary infection associated with rejection and its treatment. In children, however, primary BKV infection giving rise to allograft dysfunction may occur [156].

The definitive diagnosis of BKV nephropathy requires renal biopsy. Histopathologic features include severe tubular injury with cellular enlargement, marked nuclear atypia, epithelial necrosis, denudation of tubular basement membranes, focal intratubular neutrophilic infiltration, and mononuclear interstitial infiltration, with or without concurrent tubulins [153]. This constellation of histologic features, particularly severe tubulitis, is often misinterpreted as rejection, even by the experienced pathologist. The presence of well-demarcated basophilic or amphophilic intranuclear viral inclusions, primarily within the tubular and parietal epithelium of the Bowman capsule, can help distinguish BKV disease from rejection. Additional tests such as immunohistochemistry, PCR analysis, or electron microscopy of biopsied tissue aimed at the identification of BKV may be required. PCR assays of viral load in tubular cells have been reported to be a sensitive marker for diagnosis and monitoring.

Other Implications of BK Virus in Renal Transplantation BKV infection may cause ureteral obstruction due to ureteral ulceration and stenosis at the ureteric anastomosis. BKV-associated ureteral stenosis has been reported in $3 \%$ of renal transplant patients and usually occurs between 50 and 300 days after transplantation. Ulceration due to inflammation, proliferation of the transitional epithelial cells, and smooth muscle proliferation may lead to partial or total obstruction. Highlevel BKV replication is implicated in acute, late-onset, long-duration hemorrhagic cystitis after bone marrow transplantation [157]. 
There are two case reports in children of renal carcinomas arising in the transplanted kidney in association with BK virus nephropathy. It remains unclear whether BK virus itself has oncogenic potential in the transplant setting, but this is possible given that the big T antigen (T-Ag) expressed by polyomavirus family viruses has been shown to have the ability to disrupt chromosomal integrity $[158,159]$.

Treatment Whether patients with asymptomatic viremia or viruria need specific therapeutic intervention is not certain. Review of the literature suggests that careful reduction of immune suppression, combined with active surveillance for rejection, will result in clinical improvement. Reduction in immunosuppression may precipitate episodes of acute cellular rejection, which need to be judiciously treated with corticosteroids. The outcome of BKV nephropathy is unpredictable, and stabilization of renal function may occur regardless of whether maintenance immunotherapy is altered or not [160].

Cyclosporin, mycophenolate, and sirolimus have all been shown to possess in vitro antiviral activity against BK virus, but these findings have not been confirmed by in vivo clinical trials. Several studies have sought to identify a particular immunosuppressant or combination of drugs that increases the risk of BK virus, but results have not favored one particular regimen over another [154]. A systematic review by Johnston et al. in adults found that there was no reduction in graft loss by combining cidofovir or leflunomide with immunosuppression reduction to treat polyomavirusassociated nephropathy [161]. Prospective trials are required to address the impact of various immunosuppressive agents on BK virus replication, and randomized controlled trials are still required to define the optimal treatment of the condition.

\section{Viral Hemorrhagic Fever}

Viral hemorrhagic fever involves at least 12 distinct RNA viruses that share the propensity to cause severe disease with prominent hemorrhagic manifestations. The viral hemorrhagic fevers, widely distributed throughout both temperate and tropical regions of the world, are important causes of mortality and morbidity in many countries. Most viral hemorrhagic fevers are zoonoses (with the possible exception of dengue virus), in which the virus is endemic in animals and human infection is acquired through the bite of an insect vector. Aerosol and nosocomial transmissions from infected patients are important for Lassa, Junin, Machupo, and Congo-Crimean hemorrhagic fevers and Marburg and Ebola viruses.

Viral hemorrhagic fevers have many clinical similarities but also important differences in their severity, major organs affected, prognosis, and response to treatment. In all viral hemorrhagic fevers, severe cases occur in only a minority of those affected; subclinical infection or nonspecific febrile illness occurs in the majority. Fever, myalgia, headache, conjunctival suffusion, and erythematous rash occur in all the viral hemorrhagic fevers [162]. Hemorrhagic manifestations range from petechiae and bleeding from venipuncture sites to severe hemorrhage into the GI tract, kidney, and other organs. A capillary leak syndrome, with evidence of hemoconcentration, pulmonary edema, oliguria, and ultimately shock, occurs in the most severely affected patients. Renal involvement occurs in all the viral hemorrhagic fevers, proteinuria is common, and prerenal failure is seen in all severe cases complicated by shock. However, in Congo-Crimean hemorrhagic fever and hemorrhagic fever with renal syndrome (HFRS), an interstitial nephritis, which may be hemorrhagic, is characteristic, and renal impairment is a major component of the illness. 


\section{Dengue}

Dengue is caused by a flavivirus that is endemic and epidemic in tropical America, Africa, and Asia, where the mosquito vector Aedes aegypti is present [163]. Classic dengue is a self-limited nonfatal disease; dengue hemorrhagic fever and dengue shock syndrome, which occur in a minority of patients, have a high mortality if not aggressively treated with fluids. After an incubation period of 5-8 days, the illness begins with fever, headache, arthralgia, weakness, vomiting, and hyperesthesia. In uncomplicated dengue, the fever usually lasts 5-7 days. Shortly after onset, a maculopapular rash appears, sparing the palms and the soles, and is occasionally followed by desquamation. Fever may reappear at the onset of the rash.

In dengue hemorrhagic fever and dengue shock syndrome, the typical febrile illness is complicated by hemorrhagic manifestations, ranging from a positive tourniquet test result or petechiae to purpura, epistaxis, and GI bleeding with thrombocytopenia and evidence of a consumptive coagulopathy. Increased capillary permeability is suggested by hemoconcentration, edema, and pleural effusions [163]. In severe cases, hypotension and shock supervene, largely as a result of hypovolemia. Renal manifestations include oliguria, proteinuria, hematuria, and rising urea and creatinine. AKI occurs in patients with severe shock, primarily as a result of renal underperfusion. However, glomerular inflammatory changes may also occur. Children with dengue hemorrhagic fever show hypertrophy of endothelial and mesangial cells, mononuclear cell infiltrate, thinning of basement membranes, and deposition of $\operatorname{IgG}, \operatorname{IgM}$, and $\mathrm{C} 3$. Electron microscopy shows viral particles within glomerular mononuclear cells [164].

The diagnosis of dengue is made by isolation of the virus from blood or by serologic testing. There is no specific antiviral treatment, and management of patients with dengue shock syndrome or dengue hemorrhagic fever depends on aggressive circulatory support and volume replacement with colloid and crystalloid [165,166]. With correction of hypovolemia, renal impairment is usually reversible, but dialysis may be required in patients with established AKI.

\section{Yellow Fever}

Yellow fever is caused by a flavivirus and is transmitted by mosquito bites, typically Aedes species. It remains an important public health problem in Africa and South America. Renal manifestations are common and include albuminuria and oliguria. Over the next few days after first manifestation of infection, shock, delirium, coma, and renal failure develop, and death occurs 7-10 days after onset of symptoms. Laboratory findings include thrombocytopenia and evidence of hemoconcentration, rising urea and creatinine levels, hypernatremia, and deranged liver function test results. Pathologic findings include necrosis of liver lobules, cloudy swelling and fatty degeneration of the proximal renal tubules, and, often, petechiae in other organs. The oliguria appears to be prerenal and is due to hypovolemia; later, acute tubular necrosis supervenes. At present, there is no effective antiviral agent for yellow fever.

\section{Congo-Crimean Hemorrhagic Fever (CCHF)}

Congo-Crimean hemorrhagic fever, first recognized in the Soviet Union, is now an important human disease in Eastern Europe, Asia, and Africa. It is a tick-borne zoonotic viral disease caused by CCHF virus of the genus Nairovirus (family Bunyaviridae) [167]. Severely affected patients become stuporous or comatose 5-7 days into the illness, with evidence of hepatic and renal failure and shock. Proteinuria and hematuria are often present. The disease is fatal in 15-50\% of cases. The WHO recommends ribavirin as the treatment of choice, but a systematic meta-analysis showed no change in mortality rate in the randomized controlled trials; observational studies showed a reduction in mortality by $44 \%$ but were heavily confounded $[167,168]$. Randomized controlled 
trials are needed in a setting with full supportive care to further address this question. The role of immunoglobulin has also been tried, but no case-control trials have been conducted to support a beneficial effect.

\section{Rift Valley Fever}

Rift Valley fever is found in many areas of sub-Saharan Africa. In humans, most infections follow mosquito bites or animal exposure. The infection may present as an uncomplicated febrile illness, with muscle aches and headaches. In $10 \%$ of patients, encephalitis or retinal vasculitis occurs as a complication. In a small proportion of cases, a fulminant and often fatal hemorrhagic illness occurs with hematemesis, melena, epistaxis, and evidence of profound DIC. Severe hepatic derangement, renal failure, and encephalopathy are often present. Despite intensive care, mortality is high.

\section{Hemorrhagic Fever and Renal Syndrome (Hantavirus)}

The viruses causing HFRS all belong to the Hantavirus genus in the Bunyaviridae family. The hantaviruses are distributed worldwide and are maintained in nature through chronic infection of rodents and small mammals [169]. Transmission to humans is by aerosolized infectious excreta. Human disease usually occurs in summer among rural populations with exposure to rodent-infested barns or grain stores. Urban transmission can occur, however. At least five hantaviruses are known to cause HFRS: Hantaan, Seoul, Puumala, Porogia, and Belgrade viruses. HFRS is endemic in a belt from Norway in the west through Sweden, Finland, the Soviet Union, China, and Korea to Japan in the east. The clinical severity of HFRS varies throughout this belt. Clinical entities include Korean hemorrhagic fever, nephropathia epidemica in Scandinavia, and epidemic hemorrhagic fever in Japan and China.

In general, HFRS due to Hantaan, Porogia, and Belgrade viruses is more severe and has higher mortality than that due to Puumala virus (nephropathia epidemica) or Seoul virus. Hantaan is predominant in the Far East, Porogia and Belgrade in the Balkans, and Puumala in Western Europe; Seoul has a worldwide distribution. The clinical features of the disease vary [170]. The incubation period is 4-42 days. Although HFRS occurs with the same clinical picture in children as in adults, both incidence rates and antibody prevalence rates are very low in children under 10 years of age. Mild cases are indistinguishable from other febrile illnesses. In more severe cases, fever, headache, myalgia, abdominal pain, and dizziness are associated with the development of periorbital edema, proteinuria, and hematuria. There is often conjunctival injection, pharyngeal injection, petechiae, and epistaxis or GI bleeding. The most severely affected patients develop shock and renal failure. The disease usually passes through five phases: febrile, hypotensive, oliguric, diuretic, and convalescent. Laboratory findings include anemia, lymphocytosis, thrombocytopenia, prolonged prothrombin and bleeding times, and elevated levels of fibrin degradation products. Liver enzyme levels are elevated, and urea and creatinine levels are elevated during the oliguric phase. Proteinuria and hematuria are consistent findings. The renal histopathologic findings are those of an interstitial nephritis with prominent hemorrhages in the renal medullary interstitium and renal cortex. Acute tubular necrosis may also be seen. Immunohistochemical analysis reveals deposition of IgG and C3, and the GBM, mesangial, and subendothelial deposits may be seen on electron microscopy [171].

Recovery from Hantavirus-associated disease is generally complete, although chronic renal insufficiency is a rare sequel of HFRS. In mildly affected patients, the disease is self-limiting, and spontaneous recovery occurs. However, in severe cases, with shock, bleeding, and renal failure, dialysis and intensive circulatory support may be required. Mortality rates vary depending on the strain of virus; rates are 5-15\% for hemorrhagic fever and renal syndrome in China and significantly lower for the milder Finnish form associated with the Puumala virus strain. 
Ribavirin is active against Hantaan viruses in vitro, and clinical trials indicate that both mortality and morbidity can be reduced by treatment with this antiviral agent if it is administered early in the course of illness. Dosages of $33 \mathrm{mg} / \mathrm{kg}$ followed by $16 \mathrm{mg} / \mathrm{kg}$ every $6 \mathrm{~h}$ for 4 days and then $8 \mathrm{mg} / \mathrm{kg}$ every $8 \mathrm{~h}$ for 3 days have been used [172].

\section{Lassa Fever}

Lassa fever is a common infection in West Africa, caused by an arenavirus, and usually manifests as a nonspecific febrile illness. In $10 \%$ of cases, a fulminant hemorrhagic disease occurs. In severe cases, proteinuria and hematuria are usually present, and renal failure may occur. Ribavirin is effective in decreasing mortality. As in other hemorrhagic fevers, intensive hemodynamic support and correction of the hemostatic derangements are important components of therapy [162].

\section{Argentine and Bolivian Hemorrhagic Fevers}

Junin and Machupo viruses, the agents of Argentine and Bolivian hemorrhagic fever, respectively, cause hemorrhagic fevers with prominent neurologic features and systemic and hemorrhagic features similar to those of Lassa fever. Oliguria, shock, and renal failure occur in the most severe cases.

\section{Marburg Disease and Ebola Virus Disease}

Marburg and Ebola viruses have been associated with outbreaks of nosocomially transmitted hemorrhagic fever. West Africa has experienced the largest outbreak of Ebola, with several thousand cases in Liberia, Sierra Leone, and Guinea. Mortality in this outbreak has been high with 40-60 \% of those affected succumbing. Both viruses cause fulminant hemorrhagic fever. Onset is with high fever, headache, sore throat, myalgia, and profound prostration. An erythematous rash on the trunk is followed by hemorrhagic conjunctivitis, bleeding, impaired renal function, shock, and respiratory failure. Renal histopathologic findings in fatal cases are of tubular necrosis, with fibrin deposition in the glomeruli. There is no specific treatment for these disorders.

\section{Other Common Virus Infections}

There are many ubiquitous viral pathogens that infect large proportions of the population annually and yet are rarely associated with renal disease. The literature contains scattered reports of acute nephritis after infection with many of these viruses. The improvement in molecular diagnostic techniques has led to the recognition of other viruses that play an important role in childhood illness, for example, the human metapneumovirus [213] and Boca virus [214]. The latter only appears to have the greatest clinical impact when present in combination with other viruses. There are no reports of a significant direct renal pathology with these viruses.

Adenovirus. Adenovirus is a major cause of hemorrhagic cystitis and is commonly implicated as the cause of hemorrhagic cystitis in [173]. Boys are affected more often than girls, and hematuria persists for 3-5 days. Microscopic hematuria, dysuria, and frequency may occur for longer periods. Adenovirus types 11 and 21 are the usual strains isolated. It has been implicated in causing necrotizing granulomatous tubulointerstitial nephritis in transplant recipients, primarily affecting the distal nephron $[174,175]$. The prevalence of viremia in adult transplant recipients is estimated at $6.5 \%$. Case reports in adults have reported treatment with immunosuppressant reduction, intravenous cidofovir, and immunoglobulin to try and prevent rejection [175].

Enterovirus. Picornaviruses, including enteroviruses, have been linked with acute nephritis and AKI associated with rhabdomyolysis. Coxsackie B virus can be isolated in urine. Direct infection of kidney cells is supported by in vitro work demonstrating lytic infection of human podocyte and proximal tubular epithelial cell cultures, although different strains exhibit variable degrees of 
nephrotropism. Renal damage in vivo may have both a direct lytic mechanism and an immunecomplex basis [176]. In the newborn, enteroviruses cause fulminant disease with DIC, shock, and liver failure, and AKI may occur.

Influenza. Influenza A viruses are important infectious agents that have caused pandemics over the last two decades. The beginning of the last decade saw outbreaks of Avian flu $\left(\mathrm{H}_{5} \mathrm{~N}_{1}\right.$ and $\left.\mathrm{H}_{7} \mathrm{~N}_{7}\right)$, and the latter part of the decade dealt with the pandemic of $\mathrm{H}_{1} \mathrm{~N}_{1}$. Both cause a flulike illness with prominent respiratory and gastrointestinal symptoms. Renal failure can develop as part of the critical illness, in particular renal tubular necrosis. The WHO recommends that a standard regimen of oseltamivir used to treat seasonal flu be used to treat $\mathrm{H}_{5} \mathrm{~N}_{1}$, but in the severely ill higher doses may be required [177, 178]. Oseltamivir-resistant viruses have been reported in Southeast Asia. Peramivir and zanamivir have also been used to treat influenza A virus subtypes. Combinations of antiviral drugs with different modes of action have been explored to improve the outcome of influenza viremia, and studies are still underway in this area [177].

Measles Virus. Renal involvement from measles virus is uncommon, although measles virus can be cultured from the kidney in fatal cases. An acute GN has been reported to follow measles with evidence of immune deposits containing measles virus antigen within the glomeruli. The nephritis is generally self-limiting [179].

Mumps Virus. Mild renal involvement is common during the acute phase of mumps infection. One-third of children with mumps have abnormal urinalysis results, with microscopic hematuria or proteinuria. Mumps virus may be isolated from urine during the first 5 days of the illness, at a time when urinalysis findings are abnormal. Plasma creatinine concentrations usually remain normal, despite the abnormal urine sediment, but more severe cases in unvaccinated children have resulted in fatal interstitial nephritis with interstitial mononuclear cell infiltration, edema, and focal tubular epithelial cell damage [180]. Renal biopsy specimens in adult mumps nephritis demonstrate an MPGN with deposition of IgA, IgM, C3, and mumps virus antigen in the glomeruli, which suggests an immune-complex-mediated process [181].

\section{Coronavirus}

Most coronavirus infections are mild respiratory infections with no renal involvement. Two highly pathogenic species are described, with renal cell tropism, and the renal effects of infection require further elucidation.

SARS-CoV. Severe acute respiratory syndrome (SARS) was first seen in South China in 2002. It is caused by a SARS coronavirus (SARS-CoV). Predominantly, it causes a viral pneumonia, with diffuse alveolar damage; it has considerable mortality [182]. Renal effects are not generally significant in the pathophysiology of SARS. Acute renal impairment is uncommon in SARS but where present is associated with a high mortality and is a negative prognostic indicator for survival [183]. Case reports have documented rhabdomyolysis in association with SARS and AKI as a cause for AKI. SARS-CoV has been found in the kidney tissue at postmortem [184, 185]. SARS-CoV enters cells via angiotensin-converting enzyme 2 (ACE2) [186], and it is thought that the invasion of the kidney tissue reflects the virus' tropism for ACE2, which is expressed on kidney cells.

Middle East Region Coronavirus (MERS-CoV). MERS-CoV causes an illness clinically similar to SARS-CoV. It was discovered following an outbreak in the Middle East in 2012, and new cases continue to arise. Mortality is high - up to $40 \%$. AKI has been noted in a number of case reports. In vitro studies suggest that MERS-CoV has tropism for kidney epithelial cells [187]. 


\section{Parasitic Infections}

Chronic exposure to infectious agents is a major factor in the increased prevalence of glomerular diseases in developing countries. Malaria is the best-documented parasitic infection associated with glomerular disease, but other parasitic infections including schistosomiasis, filariasis, leishmaniasis, and possibly helminth infections may also induce nephritis or nephrosis.

\section{Malaria}

\section{Plasmodium malariae and Quartan Malaria Nephropathy}

Malaria is estimated to cause up to 500 million clinical cases of illness and more than one million deaths each year. The association of $P$. malariae (quartan) malaria and nephritis has been well known in both temperate and tropical zones since the end of the nineteenth century.

Epidemiologic studies early evidence for a role of Plasmodium malariae in glomerular disease. Chronic renal disease was a major cause of morbidity and mortality in British Guiana in the 1920s. The frequent occurrence of $P$. malariae in the blood of these patients led to detailed epidemiologic studies that implicated malaria as a cause of the nephrosis. After the eradication of malaria from British Guiana, chronic renal disease ceased to be a major cause of death in that country [188].

The link between malaria and nephrotic syndrome was strengthened by studies in West Africa in the 1950s and 1960s that demonstrated a high prevalence of nephrotic syndrome in the Nigerian population [189]. The pattern of nephrotic syndrome differed from that in temperate climates, with an older peak age, extremely poor prognosis, and unusual histologic features. The incidence of P. malariae parasitemia in patients with the nephrotic syndrome in Nigeria was vastly in excess of that occurring in the general population, whereas the incidence of Plasmodium falciparum parasitemia was similar to that in the general population. The age distribution of nephrotic syndrome also closely paralleled that of $P$. malariae infection [189]. In some affected patients, circulating immune complexes and immunoglobulin, complement, and antigens were present in the glomeruli that were recognized by $P$. malariae-specific antisera.

\section{Clinical and Histopathologic Features of Quartan Malaria Nephropathy}

Most patients have poorly selective proteinuria and are unresponsive to treatment with steroids or immunosuppressive agents. The characteristic lesions of quartan nephropathy are capillary wall thickening and segmental glomerular sclerosis, which lead to progressive glomerular changes and secondary tubular atrophy [189]. Cellular proliferation is conspicuously absent. Electron microscopy shows foot-process fusion, thickening of the basement membrane, and increase in subendothelial basement membrane-like material. Immunofluorescent studies show granular deposits of immunoglobulin, complement, and P. malariae antigen in approximately one-third of patients. The prognosis for the nephrotic syndrome in most African studies has been poor, regardless of whether the histologic findings were typical of quartan malaria nephropathy or whether P. malariae parasitemia was implicated. Treatment with steroids and azathioprine is generally ineffective, and a significant proportion of patients progress to renal failure.

In addition to the histologic pattern, termed quartan malaria nephropathy, $P$. malariae infection is associated with a variety of other forms of histologic appearance, including proliferative GN and MGN.

Although quartan malaria nephropathy has been clearly linked to P. malariae infection in Nigeria, studies from other regions in Africa have not revealed the typical histopathologic findings described in the Nigerian studies. Furthermore, quartan malaria nephropathy may be seen in children with no evidence of $P$. malariae infection or deposition of malaria antigens in the kidney. This, together with 
the fact that antimalarial treatment does not affect the progression of the disorder, raises the possibility that factors other than malaria might be involved in the initiation and perpetuation of the disorder. There is now a view that the patterns of childhood renal disease described in the last century may no longer be representative of the current situation. The variable patterns of renal disease throughout Africa may no longer reflect a dominant role for "malarial glomerulopathy," and the relative causative role of tropical infections in nephropathy remains an unanswered question [190]. Most likely, a number of different infectious processes, including malaria, hepatitis B, schistosomiasis, and perhaps other parasitic infections that cause chronic or persistent infections and often occur concurrently in malaria areas, may all result in glomerular injury and a range of overlapping histopathologic features.

\section{Renal Disease Associated with Plasmodium falciparum Infection}

Plasmodium falciparum appears to be much less likely to cause a significant glomerular pathology. Epidemiologic studies have failed to show a clear association between parasitemia and the nephrotic syndrome. Whereas renal failure appears to be a common complication of severe malaria in adults, it seldom occurs in children.

Renal biopsy specimens from adult patients with acute $P$. falciparum infections who have proteinuria or hematuria show evidence of glomerular changes, including hypercellularity, thickening of basement membranes, and hyperplasia and hypertrophy of endothelial cells [191]. Electron microscopy reveals electron-dense deposits in the subendothelial and paramesangial areas. Deposits of IgM, with or without IgG, are localized mainly in the mesangial areas. Plasmodium falciparum antigens can be demonstrated in the mesangial areas and along the capillary wall, which suggests an immune-complex GN. The changes, generally mild and transient, are probably unrelated to the AKI that may complicate severe $P$. falciparum infection [191]. Heavily parasitized erythrocytes play a central role in the various pathologic factors [192].

Renal failure occurring in severe $P$. falciparum malaria is usually associated with acidosis, volume depletion, acute intravascular hemolysis, or heavy parasitic infection that leads to acute tubular necrosis. Recent studies have confirmed an important role for volume depletion in children with severe Plasmodium falciparum malaria, who characteristically have evidence of tachycardia, tachypnea, poor perfusion, and in severe cases hypotension [193]. Volume expansion with either colloid or crystalloid results in improvement in hemodynamic indices and reduction in acidosis [194]. However, a phase 3 randomized trial of $20-40 \mathrm{ml} / \mathrm{kg}$ albumin, saline, or maintenance fluids only showed increased mortality in patients receiving bolus fluids [195]. This surprising finding has been intensively debated, and bolus fluids have been part of standard resuscitation measures for children with critical illness worldwide. A likely explanation for the adverse affects of fluid bolus is that deterioration in pulmonary or neurological condition was associated with fluid administration. As the trial was conducted in African countries where ventilator support was not available, it is not clear that the findings can be extrapolated to settings where ventilator support and modern intensive care are available. However in the light of this trial, routine volume expansion with colloid or crystalloid is currently not recommended for children with severe malaria in settings where intensive care monitoring and support is not available.

\section{Blackwater Fever}

The term blackwater fever refers to the combination of severe hemolysis, hemoglobinuria, and renal failure. It was more common at the start of the twentieth century in nonimmune individuals receiving intermittent quinine therapy for P. falciparum malaria. Blackwater fever has become rare since 1950, when quinine was replaced by chloroquine. However, the disease reappeared in the 1990s, after the 
increase in use of quinine because of the development of chloroquine-resistant organisms. Since then, several cases have been described after therapy with halofantrine and mefloquine, molecules similar to quinine (amino alcohol family) [196]. Renal failure generally occurred in the context of severe hemolytic anemia, hemoglobinuria, and jaundice. The pathophysiology of the disorder is unclear; however, it appears that a double sensitization of the red blood cells to the $P$. falciparum and to the amino alcohols is necessary to provoke the hemolysis. Histopathologic findings include swelling and vacuolization of proximal tubules, necrosis and degeneration of more distal tubules, and hemoglobin deposition in the renal tubules. Recent studies indicate a better outcome with earlier initiation of intensive care and dialysis combined with necessary changes in antimalarial medications.

\section{Schistosomiasis}

Schistosomiasis affects 200 million people living in endemic areas of Asia, Africa, and South America. The infection is usually acquired in childhood, but repeated infections occur throughout life. Schistosoma japonicum is found only in the Orient, whereas Schistosoma haematobium occurs throughout Africa, the Middle East, and areas of Southwest Asia. Schistosoma mansoni is widespread in Africa, South America, and Southwest Asia.

Human infection begins when the cercarial forms invade through the skin, develop into schistosomula, and move to the lungs via the lymphatics or blood. They then migrate to the liver and mature in the intrahepatic portal venules, where male/female pairing takes place. The adult worm pairs then migrate to their final resting site - the venules of the mesenteric venous system of the large intestine (S. mansoni) or in the venules of the urinary tract (S. haematobium). The females release large numbers of eggs, which may remain embedded in the tissues, embolize to the liver or lungs, or pass into the feces or urine.

Clinical manifestations may occur at any stage of the infection. Cercarial invasion may cause an intense itchy papular rash. Katayama fever is an acute serum sickness-like illness that occurs several weeks after infection, as eggs are being deposited in the tissues. Deposition of the eggs in tissues results in inflammation of the intestines, fibrosis of the liver, and portal hypertension. With S. haematobium, chronic inflammation and fibrosis of the ureters and bladder may lead to obstructive uropathy.

Renal manifestations of schistosomiasis occur most commonly in S. mansoni infection [197]. Schistosomal nephropathy usually presents with symptoms including granulomatous inflammation in the ureters and bladder, but glomerular disease (probably on an immune-complex basis) may also occur. Renal disease usually occurs in older children or young adults with long-term infection, but serious disease may also occur in young children. There have been isolated case reports of young children presenting with nephrotic syndrome secondary to Schistosoma haematobium infection directly causing membranoproliferative glomerulonephritis [198].

The early renal tract manifestations of schistosomiasis are suprapubic discomfort, frequency, dysuria, and terminal hematuria. In more severe cases, evidence of urinary obstruction appears. Poor urinary stream, straining on micturition, a feeling of incomplete bladder emptying, and a constant urge to urinate may be severely disabling symptoms. The fibrosis and inflammation of ureters, urethra, and bladder may be followed by calcification and may result in hydroureter, hydronephrosis, and bladder neck obstruction. Renal failure may ultimately develop, and there is a suspicion that squamous cell carcinoma of the bladder may be linked to the chronic infective and inflammatory process. Secondary bacterial infection is common within the obstructed and inflamed urinary tract.

The hepatosplenic form of $S$. mansoni infection may be accompanied by a glomerulopathy in $12-15 \%$ of cases, manifested in the majority as nephrotic syndrome. Histopathologic findings include mesangioproliferative GN, focal segmental glomerulosclerosis, mesangiocapillary GN, 
MGN, and focal segmental hyalinosis. Immune complexes may be detected in the circulation of these patients, and glomerular granular deposition of IgM, C3, and schistosomal antigens are seen on immunofluorescence [197]. Usually schistosoma-specific nephropathy is a progressive disease and is not influenced by antiparasitic or immunosuppressive therapy.

The diagnosis is confirmed by the detection of schistosomula eggs in feces, urine, or biopsy specimens. Eggs are shed into the urine with a diurnal rhythm, and urine collected between $11 \mathrm{AM}$ and $1 \mathrm{PM}$ is the most useful. Urinary sediment obtained by centrifugation or filtration through a Nuclepore membrane should be examined. In cases in which studies of urine and feces yield negative results in patients in whom the diagnosis is suspected, rectal biopsy specimens taken approximately $9 \mathrm{~cm}$ from the anus have a high diagnostic yield for both $S$. mansoni and $S$. haematobium infection. Biopsy of the liver or bladder may be required to establish the diagnosis. Antibodies indicating previous infection can be detected using enzyme-linked immunosorbent assay or radioimmunoassay. The tests are sensitive but lack specificity and may not differentiate between past exposure and current infection.

Praziquantel is the drug of choice for treatment of schistosomiasis. A single oral dose of $40 \mathrm{mg} / \mathrm{kg}$ is effective in $S$. haematobium and $S$. mansoni infection and is usually well tolerated. The alternative drug for $S$. mansoni infection is oxamniquine. Complete remission of urinary symptoms may occur in renal disease of short duration, but in late disease with extensive fibrosis, scarring, and calcification, obstructive uropathy and renal failure may persist after the infection has been eradicated.

\section{Leishmaniasis}

Visceral leishmaniasis is a chronic protozoon infection characterized by fever, hepatosplenomegaly, anemia, leukopenia, and hyperglobulinemia [199]. Proteinuria and/or microscopic hematuria or pyuria has been reported in $50 \%$ of patients with visceral leishmaniasis. AKI in association with interstitial nephritis has also been reported. Renal histologic analysis in patients with visceral leishmaniasis reveals glomerular changes, with features of a mesangial proliferative GN or a focal proliferative GN, or a generalized interstitial nephritis with interstitial edema, mononuclear cell infiltration, and focal tubular degeneration. Immunofluorescence reveals deposition of $\operatorname{IgG}, \operatorname{IgM}$, and $\mathrm{C} 3$ within the glomeruli, as well as electron-dense deposits in the basement membrane and mesangium on electron microscopy [200]. Circulating immune complexes together with immunoglobulin and complement deposition in the glomeruli suggests an immune-complex cause. Renal disease in leishmaniasis is usually mild and may resolve after treatment of the infection. Renal dysfunction may be associated with treatment for visceral leishmaniasis with antimony compounds.

\section{Filariasis}

Proteinuria is more common in filarial hyperendemic regions of West Africa than in nonfilarial areas. Renal histologic analysis has shown a variety of different histopathologic appearances; the most common is diffuse mesangial proliferative GN with C3 deposition in the glomeruli [201]. Renal biopsy specimens also demonstrate large numbers of eosinophils in the glomeruli, and microfilariae may be seen in the lumen of glomerular capillaries. Filarial antigens have been detected within immune deposits within the glomeruli. Lymphatic filariasis is also associated with chyluria [202].

\section{Hydatid Disease}

Echinococcus granulosus causes chronic cysts within a variety of organs [203]. In addition, nephrotic syndrome in association with hydatid disease has been reported. Membranous nephropathy, minimal change lesions, and mesangiocapillary GN have been described in association with hydatid disease [204]. Immunofluorescence reveals deposits of immunoglobulin, complement, and 
hydatid antigens within the glomeruli. Remission of nephrotic syndrome has been reported with treatment by antiparasitic agents such as albendazole.

\section{Toxoplasmosis}

Nephrotic syndrome has occasionally been reported as a manifestation of congenital toxoplasmosis.

Dissemination of previously latent toxoplasma infection in patients undergoing treatment with immunosuppressive drugs has been increasingly recognized in recent years. Reactivation of toxoplasmosis or progression of recently acquired primary infection should be considered in patients undergoing renal transplantation or immunotherapy for renal disease who develop unexplained inflammation of any organ.

\section{Fungal Infections}

Fungal infections of the kidneys and urinary tract occur most commonly as part of systemic fungal infections in patients with underlying immunodeficiency, as focal urinary tract infections in patients with obstructive lesions, or as a result of indwelling catheters [205]. Although candidal infection is the most common fungal infection in both immunocompromised and nonimmunocompromised hosts, virtually all other fungal pathogens may invade the renal tract during severe immunocompromise.

Urinary infection with Candida albicans is most commonly a component of systemic candidiasis in patients who are severely immunocompromised. Systemic candidiasis is also seen in premature and term infants with perinatally acquired invasive candidiasis. Presentation is usually with systemic sepsis, fever or hypothermia, hepatosplenomegaly, erythematous rash, and thrombocytopenia. Systemic candidiasis may be seen on ophthalmologic investigation as microemboli in the retina. The first clue to the underlying diagnosis may be the presence of yeasts in the urine.

Candidal involvement of the urinary tract may affect all structures including the glomeruli, tubules, collecting system, ureters, and bladder. Microabscesses may form within the renal parenchyma, and large balls of fungi may completely obstruct the urinary tract at any level. AKI caused by systemic candidiasis or obstruction of the renal tracts with fungal hyphae is a well-recognized complication of systemic candidal infection. Indwelling catheters (which form a nidus for persistent infection) should be removed [205]. Successful treatment of nonobstructing bilateral renal fungal balls by fluconazole either alone or in combination with liposomal amphotericin B has been reported [206]. In the presence of obstruction, however, percutaneous nephrostomy to relieve the obstruction with anterograde amphotericin B irrigation, coupled with systemic antifungal therapy, is the mainstay of treatment. Amphotericin B is the most effective antifungal agent, but it is not excreted in the urine. Local irrigation via nephrostomy provides good results, however. For treatment of urinary tract candidiasis, it is usually combined with fluconazole or 5-flucytosine, both of which are excreted in high concentrations in the urine. Treatment is required for weeks to months to ensure complete elimination of the fungus, and the ultimate outcome is largely dependent on whether there is a permanent defect in immunity. 


\section{Miscellaneous Conditions}

\section{Hemorrhagic Shock and Encephalopathy}

In 1983, Levin et al. first described hemorrhagic shock and encephalopathy, which appeared to be distinct from previously recognized pediatric disorders [207]. Other cases have subsequently been reported from several centers in the United Kingdom, Europe, Israel, the United States, and Australia, and the syndrome is now recognized as a distinct severe childhood disorder.

Hemorrhagic shock and encephalopathy usually affect infants in the first year of life, with a peak onset at 3-4 months of age. A prodromal illness with fever, irritability, diarrhea, or upper respiratory infection occurs 2-5 days before the onset in two-thirds of cases. Affected infants develop profound shock, coma, convulsions, bleeding and evidence of DIC, diarrhea, and oliguria. Laboratory findings include acidosis, falling hemoglobin and platelet levels, elevated urea and creatinine levels, and elevated levels of hepatic transaminases. Despite vigorous intensive care, the prognosis is poor, and most affected infants die or are left severely neurologically damaged [208]. A small number of patients have been reported to survive without residual sequelae.

The renal impairment appears to be largely prerenal in origin, and when aggressive volume replacement and treatment of the shock results in improved renal perfusion, rapid improvement in renal function is usually observed. In patients with profound shock unresponsive to initial resuscitation, vasomotor nephropathy supervenes and dialysis may be required. Myoglobinuria in association with hemorrhagic shock and encephalopathy has been reported.

\section{Kawasaki Disease}

Following the description of the mucocutaneous lymph node syndrome by Kawasaki in 1968, Kawasaki disease (KD) has been recognized as a common and serious childhood illness with a worldwide distribution. Although the etiology remains unknown, epidemiologic features clearly suggest an infective cause. The disease occurs in epidemics, and wavelike spread has been demonstrated during outbreaks in Japan (see chapter " $\vee$ Vasculitis"). The main complication of $\mathrm{KD}$ is the development of a vasculitis affecting the small- and medium-sized muscular arteries. Although the coronary arteries are most commonly affected in severe cases, the vasculitis can be widespread and affect axillary, femoral, carotid, renal, and mesenteric arteries [209].

Renal involvement in KD is common, with proteinuria and sterile pyuria being commonly detected during the acute disease [210]. More severe kidney involvement is less common, but there are reports of a broad spectrum of other kidney involvement including acute kidney injury, interstitial nephritis, immune-complex nephritis, nephrotic syndrome, and hemolytic uremic syndrome. The vasculitis of KD involves neutrophil and lymphocytic influx into the vessel wall, with destruction of the intima and media. Involvement of extrarenal or intrarenal vasculature can result in areas of infarction or in hypertension.

Reported histological features in KD patients with more severe forms of renal involvement include interstitial infiltration of leukocytes, immune-complex nephritis, swelling of endothelial and mesangial cells, dense deposits, and vasculitis [210]. Treatment of KD is aimed at rapid cessation of the inflammatory process. In patients who do not respond to intravenous immunoglobulin, addition of other anti-inflammatory agents including steroids, anti-TNF agents, anakinra, or cyclosporine has been reported to be beneficial.

\section{Xanthogranulomatous Pyelonephritis (XGP)}

XGP is a chronic inflammatory disorder of the kidney that invades the parenchyma, characterized by granulomatous inflammation with giant cells and foamy histiocytes. Most are unilateral and are 
caused by urinary tract obstruction, infection, nephrolithiasis, diabetes, and/or immunocompromise. It is rare in children, but when it occurs, it typically affects boys under 8 years of age [211]. A case series by Quinn et al. showed an incidence of 6/1,000 surgically proven chronic pyelonephritis. Case reports have associated it with renal vein thrombi [212]. It presents with anorexia, weight loss, fever, chills, and dull, persistent flank pain. It occurs in $1 \%$ of all renal infections, and E. coli, Proteus, and Pseudomonas infections are implicated. XGP has also been referred to as a pseudotumor as it can be radiologically and clinically indistinguishable from renal carcinoma. The treatment is surgical [211].

\section{References}

1. Basu RK, Devarajan P, Wong H, Wheeler DS. An update and review of acute kidney injury in pediatrics. Pediatr Crit Care Med. 2011;12:339-47.

2. Fortenberry JD, Paden ML, Goldstein SL. Acute kidney injury in children: an update on diagnosis and treatment. Pediatr Clin North Am. 2013;60:669-88.

3. Andreoli SP. Acute kidney injury in children. Pediatr Nephrol. 2009;24:253-63.

4. De Vriese AS. Prevention and treatment of acute renal failure in sepsis. J Am Soc Nephrol. 2003;14:792-805.

5. Cohen RI, Hassell AM, Marzouk K, Marini C, Liu SF, Scharf SM. Renal effects of nitric oxide in endotoxemia. Am J Respir Crit Care Med. 2001;164:1890-5.

6. Nadel S, Goldstein B, Williams MD, Dalton H, Peters M, Macias WL, Abd-Allah SA, Levy H, Angle R, Wang D, et al. Drotrecogin alfa (activated) in children with severe sepsis: a multicentre phase III randomised controlled trial. Lancet. 2007;369:836-43.

7. Schneider J, Khemani R, Grushkin C, Bart R. Serum creatinine as stratified in the RIFLE score for acute kidney injury is associated with mortality and length of stay for children in the pediatric intensive care unit. Crit Care Med. 2010;38:933-9.

8. Ronco C, Legrand M, Goldstein SL, Hur M, Tran N, Howell EC, Cantaluppi V, Cruz DN, Damman K, Bagshaw SM, et al. Neutrophil gelatinase-associated lipocalin: ready for routine clinical use? An international perspective. Blood Purif. 2014;37:271-85.

9. Harrison LH, Trotter CL, Ramsay ME. Global epidemiology of meningococcal disease. Vaccine. 2009;27 Suppl 2:B51-63.

10. Edmond K, Clark A, Korczak VS, Sanderson C, Griffiths UK, Rudan I. Global and regional risk of disabling sequelae from bacterial meningitis: a systematic review and meta-analysis. Lancet Infect Dis. 2010;10:317-28.

11. Couto-Alves A, Wright VJ, Perumal K, Binder A, Carrol ED, Emonts M, de Groot R, Hazelzet J, Kuijpers T, Nadel S, et al. A new scoring system derived from base excess and platelet count at presentation predicts mortality in paediatric meningococcal sepsis. Crit Care. 2013;17:R68.

12. Kumar AA, Bhaskar E, Palamaner Subash Shantha G, Swaminathan P, Abraham G. Rhabdomyolysis in community acquired bacterial sepsis-a retrospective cohort study. PLoS One. 2009;4:e7182.

13. Brandtzaeg P, van Deuren M. Current concepts in the role of the host response in Neisseria meningitidis septic shock. Curr Opin Infect Dis. 2002;15:247-52.

14. Pathan N, Hemingway CA, Alizadeh AA, Stephens AC, Boldrick JC, Oragui EE, McCabe C, Welch SB, Whitney A, O'Gara P, et al. Role of interleukin 6 in myocardial dysfunction of meningococcal septic shock. Lancet. 2004;363:203-9. 
15. Faust SN, Levin M, Harrison OB, Goldin RD, Lockhart MS, Kondaveeti S, Laszik Z, Esmon CT, Heyderman RS. Dysfunction of endothelial protein $\mathrm{C}$ activation in severe meningococcal sepsis. N Engl J Med. 2001;345:408-16.

16. Bernard GR, Vincent JL, Laterre PF, LaRosa SP, Dhainaut JF, Lopez-Rodriguez A, Steingrub JS, Garber GE, Helterbrand JD, Ely EW, et al. Efficacy and safety of recombinant human activated protein C for severe sepsis. N Engl J Med. 2001;344:699-709.

17. Angel C, Shu T, Green J, Orihuela E, Rodriquez G, Hendrick E. Renal and peri-renal abscesses in children: proposed physio-pathologic mechanisms and treatment algorithm. Pediatr Surg Int. 2003;19:35-9.

18. Todd J, Fishaut M, Kapral F, Welch T. Toxic-shock syndrome associated with phage-group-I staphylococci. Lancet. 1978;312:1116-8.

19. Chuang YY, Huang YC, Lin TY. Toxic shock syndrome in children: epidemiology, pathogenesis, and management. Paediatr Drugs. 2005;7:11-25.

20. Buchdahl R, Levin M, Wilkins B, Gould J, Jaffe P, Matthew DJ, Dillon MJ. Toxic shock syndrome. Arch Dis Child. 1985;60:563-7.

21. Todd JK, Franco-Buff A, Lawellin DW, Vasil ML. Phenotypic distinctiveness of Staphylococcus aureus strains associated with toxic shock syndrome. Infect Immun. 1984;45:339-44.

22. Marrack P, Kappler J. The staphylococcal enterotoxins and their relatives. Science (New York, NY). 1990;248:1066.

23. Stevens DL, Ma Y, Salmi DB, McIndoo E, Wallace RJ, Bryant AE. Impact of antibiotics on expression of virulence-associated exotoxin genes in methicillin-sensitive and methicillinresistant Staphylococcus aureus. J Infect Dis. 2007;195:202-11.

24. Kaneko J, Kamio Y. Bacterial two-component and hetero-heptameric pore-forming cytolytic toxins: structures, pore-forming mechanism, and organization of the genes. Biosci Biotechnol Biochem. 2004;68:981-1003.

25. Malik ZA, Litman N. Perinephric abscess caused by community-acquired methicillin resistant Staphylococcus aureus. Pediatr Infect Dis J. 2007;26:764.

26. Dumitrescu O, Choudhury P, Boisset S, Badiou C, Bes M, Benito Y, Wolz C, Vandenesch F, Etienne J, Cheung AL, et al. Beta-lactams interfering with PBP1 induce Panton-Valentine leukocidin expression by triggering sarA and rot global regulators of Staphylococcus aureus. Antimicrob Agents Chemother. 2011;55:3261-71.

27. Kambham N. Postinfectious glomerulonephritis. Adv Anat Pathol. 2012;19:338-47.

28. Rodriguez-Iturbe B, Musser JM. The current state of post streptococcal glomerulonephritis. J Am Soc Nephrol. 2008;19:1855-64.

29. Cunningham MW. Pathogenesis of group A streptococcal infections. Clin Microbiol Rev. 2000;13:470-511.

30. Popovic-Rolovic M, Kostic M, Antic-Peco A, Jovanovic O, Popovic D. Medium- and longterm prognosis of patients with acute poststreptococcal glomerulonephritis. Nephron. 1991;58:393-9.

31. Beres SB, Sesso R, Pinto SW, Hoe NP, Porcella SF, Deleo FR, Musser JM. Genome sequence of a Lancefield group C Streptococcus zooepidemicus strain causing epidemic nephritis: new information about an old disease. PLoS One. 2008;3:e3026.

32. Gnann Jr JW, Gray BM, Griffin Jr FM, Dismukes WE. Acute glomerulonephritis following group G streptococcal infection. J Infect Dis. 1987;156:411-2.

33. Adalat S, Dawson T, Hackett SJ, Clark JE. Toxic shock syndrome surveillance in UK children. Arch Dis Child. 2014;99:1078-82. 
34. Rodriguez-Nunez A, Dosil-Gallardo S, Jordan I. Clinical characteristics of children with group A streptococcal toxic shock syndrome admitted to pediatric intensive care units. Eur J Pediatr. 2011;170:639-44.

35. Shah SS, Hall M, Srivastava R, Subramony A, Levin JE. Intravenous immunoglobulin in children with streptococcal toxic shock syndrome. Clin Infect Dis. 2009;49:1369-76.

36. Edwards RJ, Taylor GW, Ferguson M, Murray S, Rendell N, Wrigley A, Bai Z, Boyle J, Finney SJ, Jones A, et al. Specific C-terminal cleavage and inactivation of interleukin- 8 by invasive disease isolates of Streptococcus pyogenes. J Infect Dis. 2005;192:783-90.

37. Mascini EM, Jansze M, Schouls LM, Verhoef J, Van Dijk H. Penicillin and clindamycin differentially inhibit the production of pyrogenic exotoxins A and B by group A streptococci. Int J Antimicrob Agents. 2001;18:395-8.

38. Zimbelman J, Palmer A, Todd J. Improved outcome of clindamycin compared with betalactam antibiotic treatment for invasive Streptococcus pyogenes infection. Pediatr Infect Dis J. 1999;18:1096-100.

39. Darenberg J, Ihendyane N, Sjölin J, Aufwerber E, Haidl S, Follin P, Andersson J, NorrbyTeglund A. Intravenous immunoglobulin $G$ therapy in streptococcal toxic shock syndrome: a European randomized, double-blind, placebo-controlled trial. Clin Infect Dis. 2003;37:333-40.

40. Kaul R, McGeer A, Norrby-Teglund A, Kotb M, Schwartz B, O'Rourke K, Talbot J, Low DE. Intravenous immunoglobulin therapy for streptococcal toxic shock syndrome-a comparative observational study. The Canadian Streptococcal Study Group. Clin Infect Dis. 1999;28:800-7.

41. Carapetis JR, Jacoby P, Carville K, Ang SJ, Curtis N, Andrews R. Effectiveness of clindamycin and intravenous immunoglobulin, and risk of disease in contacts, in invasive group a streptococcal infections. Clin Infect Dis. 2014;59:358-65.

42. Linner A, Darenberg J, Sjolin J, Henriques-Normark B, Norrby-Teglund A. Clinical efficacy of polyspecific intravenous immunoglobulin therapy in patients with streptococcal toxic shock syndrome: a comparative observational study. Clin Infect Dis. 2014;59:851-7.

43. Menon K, McNally D, Choong K, Sampson M. A systematic review and meta-analysis on the effect of steroids in pediatric shock. Pediatr Crit Care Med. 2013;14:474-80.

44. Zimmerman JJ. A history of adjunctive glucocorticoid treatment for pediatric sepsis: moving beyond steroid pulp fiction toward evidence-based medicine. Pediatr Crit Care Med. 2007;8:530-9.

45. Brunkhorst FM, Engel C, Bloos F, Meier-Hellmann A, Ragaller M, Weiler N, Moerer O, Gruendling M, Oppert M, Grond S, et al. Intensive insulin therapy and pentastarch resuscitation in severe sepsis. N Engl J Med. 2008;358:125-39.

46. Spinale JM, Ruebner RL, Kaplan BS, Copelovitch L. Update on Streptococcus pneumoniae associated hemolytic uremic syndrome. Curr Opin Pediatr. 2013;25:203-8.

47. Gasser C, Gautier E, Steck A, Siebenmann RE, Oechslin R. Hemolytic-uremic syndrome: bilateral necrosis of the renal cortex in acute acquired hemolytic anemia. Schweiz Med Wochenschr. 1955;85:905-9.

48. Seger R, Joller P, Baerlocher K, Kenny A, Dulake C, Leumann E, Spierig M, Hitzig WH. Hemolytic-uremic syndrome associated with neuraminidase-producing microorganisms: treatment by exchange transfusion. Helv Paediatr Acta. 1980;35:359-67.

49. von Vigier RO, Seibel K, Bianchetti MG. Positive Coombs test in pneumococcus-associated hemolytic uremic syndrome. A review of the literature. Nephron. 1999;82:183-4. 
50. Copelovitch L, Kaplan BS. Streptococcus pneumoniae-associated hemolytic uremic syndrome. Pediatr Nephrol. 2008;23:1951-6.

51. Waters AM, Kerecuk L, Luk D, Haq MR, Fitzpatrick MM, Gilbert RD, Inward C, Jones C, Pichon B, Reid C, et al. Hemolytic uremic syndrome associated with invasive pneumococcal disease: the United Kingdom experience. J Pediatr. 2007;151:140-4.

52. Eber SW, Polster H, Quentin SH, Rumpf KW, Lynen R. Hemolytic-uremic syndrome in pneumococcal meningitis and infection. Importance of T-transformation. Monatsschr Kinderheilkd. 1993;141:219-22.

53. Brandt J, Wong C, Mihm S, Roberts J, Smith J, Brewer E, Thiagarajan R, Warady B. Invasive pneumococcal disease and hemolytic uremic syndrome. Pediatrics. 2002;110:371-6.

54. Lai KN, Aarons I, Woodroffe AJ, Clarkson AR. Renal lesions in leptospirosis. Aust N Z J Med. 1982;12:276-9.

55. Abdulkader RC, Silva MV. The kidney in leptospirosis. Pediatr Nephrol. 2008;23:2111-20.

56. Clerke AM, Leuva AC, Joshi C, Trivedi SV. Clinical profile of leptospirosis in South Gujarat. J Postgrad Med. 2002;48:117-8.

57. De Francesco Daher E, Oliveira Neto FH, Ramirez SM. Evaluation of hemostasis disorders and anticardiolipin antibody in patients with severe leptospirosis. Rev Inst Med Trop Sao Paulo. 2002;44:85-90.

58. Kennedy ND, Pusey CD, Rainford DJ, Higginson A. Leptospirosis and acute renal failure-clinical experiences and a review of the literature. Postgrad Med J. 1979;55:176-9.

59. Srivastava RN. Acute glomerulonephritis in Salmonella typhi infection. Indian Pediatr. 1993;30:278-9.

60. Koo JW, Park SN, Choi SM, Chang CH, Cho CR, Paik IK, Chung CY. Acute renal failure associated with Yersinia pseudotuberculosis infection in children. Pediatr Nephrol. 1996;10:582-6.

61. Fukumoto Y, Hiraoka M, Takano T, Hori C, Tsuchida S, Kikawa Y, Sudo M. Acute tubulointerstitial nephritis in association with Yersinia pseudotuberculosis infection. Pediatr Nephrol. 1995;9:78-80.

62. Sato K, Ouchi K, Komazawa M. Ampicillin vs. placebo for Yersinia pseudotuberculosis infection in children. Pediatr Infect Dis J. 1988;7:686-9.

63. Karmali MA, Steele BT, Petric M, Lim C. Sporadic cases of haemolytic-uraemic syndrome associated with faecal cytotoxin and cytotoxin-producing Escherichia coli in stools. Lancet. 1983;1:619-20.

64. Latus J, Amann K, Braun N, Alscher MD, Kimmel M. Tubulointerstitial nephritis in active tuberculosis - a single center experience. Clin Nephrol. 2012;78:297-302.

65. Figueiredo AA, Lucon AM, Junior RF, Srougi M. Epidemiology of urogenital tuberculosis worldwide. Int J Urol. 2008;15:827-32.

66. Krishnamoorthy S, Gopalakrishnan G. Surgical management of renal tuberculosis. Indian J Urol. 2008;24:369-75.

67. Woods CR. Congenital syphilis-persisting pestilence. Pediatr Infect Dis J. 2009;28:536-7.

68. Gamble CN, Reardan JB. Immunopathogenesis of syphilitic glomerulonephritis. Elution of antitreponemal antibody from glomerular immune-complex deposits. $\mathrm{N}$ Engl J Med. 1975;292:449-54.

69. Cochat P, Colon S, Bosshard S, Zech P, Traeger J. Membranoproliferative glomerulonephritis and Mycoplasma pneumoniae infection. Arch Fr Pediatr. 1985;42:29-31.

70. Narita M. Pathogenesis of extrapulmonary manifestations of Mycoplasma pneumoniae infection with special reference to pneumonia. J Infect Chemother. 2010;16:162-9. 
71. Said MH, Layani MP, Colon S, Faraj G, Glastre C, Cochat P. Mycoplasma pneumoniae-associated nephritis in children. Pediatr Nephrol. 1999;13:39-44.

72. Shah A, Check F, Baskin S, Reyman T, Menard R. Legionnaires' disease and acute renal failure: case report and review. Clin Infect Dis. 1992;14:204-7.

73. Nishitarumizu K, Tokuda Y, Uehara H, Taira M, Taira K. Tubulointerstitial nephritis associated with Legionnaires' disease. Intern Med. 2000;39:150-3.

74. Woods CR. Rocky Mountain spotted fever in children. Pediatr Clin North Am. 2013;60:455-70.

75. Quigg RJ, Gaines R, Wakely Jr PE, Schoolwerth AC. Acute glomerulonephritis in a patient with Rocky Mountain spotted fever. Am J Kidney Dis. 1991;17:339-42.

76. Alvarez-Hernandez G, Murillo-Benitez C, Del Carmen Candia-Plata M, Moro M. Clinical profile and predictors of Fatal Rocky Mountain spotted fever in children from Sonora, Mexico. Pediatr Infect Dis J. 2014. Published ahead of print. doi: 10.1097/INF.0000000000000496

77. Conlon PJ, Procop GW, Fowler V, Eloubeidi MA, Smith SR, Sexton DJ. Predictors of prognosis and risk of acute renal failure in patients with Rocky Mountain spotted fever. Am J Med. 1996;101:621-6.

78. Gikas A, Kokkini S, Tsioutis C. Q fever: clinical manifestations and treatment. Expert Rev Anti Infect Ther. 2010;8:529-39.

79. Ruiz Seco MP, Lopez Rodriguez M, Estebanez Munoz M, Pagan B, Gomez Cerezo JF, Barbado Hernandez FJ. Q fever: 54 new cases from a tertiary hospital in Madrid. Rev Clin Esp. 2011;211:240-4.

80. Tolosa-Vilella C, Rodriguez-Jornet A, Font-Rocabanyera J, Andreu-Navarro $\mathrm{X}$. Mesangioproliferative glomerulonephritis and antibodies to phospholipids in a patient with acute Q fever: case report. Clin Infect Dis. 1995;21:196-8.

81. Gikas A, Kofteridis DP, Manios A, Pediaditis J, Tselentis Y. Newer macrolides as empiric treatment for acute Q fever infection. Antimicrob Agents Chemother. 2001;45:3644-6.

82. Levy RL, Hong R. The immune nature of subacute bacterial endocarditis (SBE) nephritis. Am J Med. 1973;54:645-52.

83. Majumdar A, Chowdhary S, Ferreira MA, Hammond LA, Howie AJ, Lipkin GW, Littler WA. Renal pathological findings in infective endocarditis. Nephrol Dial Transplant. 2000;15:1782-7.

84. Baltimore RS. Infective endocarditis in children. Pediatr Infect Dis J. 1992;11:907-12.

85. Rifkinson-Mann S, Rifkinson N, Leong T. Shunt nephritis. Case Rep J Neurosurg. 1991;74:656-9.

86. Vernet $\mathrm{O}$, Rilliet B. Late complications of ventriculoatrial or ventriculoperitoneal shunts. Lancet. 2001;358:1569-70.

87. Haffner D, Schindera F, Aschoff A, Matthias S, Waldherr R, Scharer K. The clinical spectrum of shunt nephritis. Nephrol Dial Transpl. 1997;12:1143-8.

88. Iwata Y, Ohta S, Kawai K, Yamahana J, Sugimori H, Ishida Y, Saito K, Miyamori T, Futami K, Arakawa Y, et al. Shunt nephritis with positive titers for ANCA specific for proteinase 3. Am J Kidney Dis. 2004;43:e11-6.

89. Chan TM. Hepatitis B and renal disease. Curr Hepat Rep. 2010;9:99-105.

90. Johnson RJ, Couser WG. Hepatitis B infection and renal disease: clinical, immunopathogenetic and therapeutic considerations. Kidney Int. 1990;37:663-76.

91. Guillevin L, Lhote F, Cohen P, Sauvaget F, Jarrousse B, Lortholary O, Noël LH, Trépo C. Polyarteritis nodosa related to hepatitis B virus. A prospective study with long-term observation of 41 patients. Medicine. 1995;74:238-53. 
92. van Timmeren MM, Heeringa P, Kallenberg CG. Infectious triggers for vasculitis. Curr Opin Rheumatol. 2014;26:416-23.

93. Guillevin L, Mahr A, Callard P, Godmer P, Pagnoux C, Leray E, Cohen P. Hepatitis B virusassociated polyarteritis nodosa: clinical characteristics, outcome, and impact of treatment in 115 patients. Medicine. 2005;84:313-22.

94. Verma R, Lalla R, Babu S. Mononeuritis multiplex and painful ulcers as the initial manifestation of hepatitis B infection. BMJ Case Rep. 2013;2013. published online. doi:10.1136/bcr2013-009666. Omit the second 2013.

95. Bhimma R, Coovadia HM. Hepatitis B virus-associated nephropathy. Am J Nephrol. 2004;24:198-211.

96. Janssen HL, van Zonneveld $M$, van Nunen AB, Niesters HG, Schalm SW, de Man RA. Polyarteritis nodosa associated with hepatitis B virus infection. The role of antiviral treatment and mutations in the hepatitis B virus genome. Eur J Gastroenterol Hepatol. 2004; 16:801-7.

97. Ren J, Wang L, Chen Z, Ma ZM, Zhu HG, Yang DL, Li XY, Wang BI, Fei J, Wang ZG, et al. Gene expression profile of transgenic mouse kidney reveals pathogenesis of hepatitis B virus associated nephropathy. J Med Virol. 2006;78:551-60.

98. Elewa U, Sandri AM, Kim WR, Fervenza FC. Treatment of hepatitis B virus-associated nephropathy. Nephron Clin Pract. 2011;119:c41-9; discussion c49.

99. Lin CY. Treatment of hepatitis B virus-associated membranous nephropathy with recombinant alpha-interferon. Kidney Int. 1995;47:225-30.

100. Shah HH, Patel C, Jhaveri KD. Complete remission of hepatitis B virus-associated nephrotic syndrome from IgA nephropathy following peginterferon therapy. Ren Fail. 2013;35:295-8.

101. Li H, Yuan X, Qiu L, Zhou Q, Xiao P. Efficacy of adefovir dipivoxil combined with a corticosteroid in 38 cases of nephrotic syndrome induced by hepatitis B virus-associated glomerulonephritis. Ren Fail. 2014;36:1404-6.

102. Armstrong GL, Wasley A, Simard EP, McQuillan GM, Kuhnert WL, Alter MJ. The prevalence of hepatitis C virus infection in the United States, 1999 through 2002. Ann Intern Med. 2006;144:705-14.

103. Jonas MM. Children with hepatitis C. Hepatology. 2002;36:S173-8.

104. Tang SC, Lai KN. Hepatitis C virus-associated glomerulonephritis. Contrib Nephrol. 2013;181:194-206.

105. Rostaing L, Izopet J, Kamar N. Hepatitis C virus infection in nephrology patients. J Nephropathol. 2013;2:217-33.

106. Stehman-Breen C, Willson R, Alpers CE, Gretch D, Johnson RJ. Hepatitis C virus-associated glomerulonephritis. Curr Opin Nephrol Hypertens. 1995;4:287-94.

107. Yamabe H, Inuma H, Osawa H, Kaizuka M, Tamura N, Tsunoda S, Fujita Y, Shiroto K, Onodera K. Glomerular deposition of hepatitis $\mathrm{C}$ virus in membranoproliferative glomerulonephritis. Nephron. 1996;72:741.

108. Batty Jr DS, Swanson SJ, Kirk AD, Ko CW, Agodoa LY, Abbott KC. Hepatitis C virus seropositivity at the time of renal transplantation in the United States: associated factors and patient survival. Am J Transplant. 2001;1:179-84.

109. Schwarz KB, Gonzalez-Peralta RP, Murray KF, Molleston JP, Haber BA, Jonas MM, Rosenthal P, Mohan P, Balistreri WF, Narkewicz MR, et al. The combination of ribavirin and peginterferon is superior to peginterferon and placebo for children and adolescents with chronic hepatitis C. Gastroenterology. 2011;140:450-8.e451. 
110. Khaderi S, Shepherd R, Goss JA, Leung DH. Hepatitis C in the pediatric population: transmission, natural history, treatment and liver transplantation. World $\mathrm{J}$ Gastroenterol. 2014;20:11281-6.

111. Beuthien W, Mellinghoff HU, Kempis J. Vasculitic complications of interferon-alpha treatment for chronic hepatitis $\mathrm{C}$ virus infection: case report and review of the literature. Clin Rheumatol. 2005;24:507-15.

112. Myerson D, Hackman RC, Nelson JA, Ward DC, McDougall JK. Widespread presence of histologically occult cytomegalovirus. Hum Pathol. 1984;15:430-9.

113. Beneck D, Greco MA, Feiner HD. Glomerulonephritis in congenital cytomegalic inclusion disease. Hum Pathol. 1986;17:1054-9.

114. Richardson WP, Colvin RB, Cheeseman SH, Tolkoff-Rubin NE, Herrin JT, Cosimi AB, Collins AB, Hirsch MS, McCluskey RT, Russell PS, et al. Glomerulopathy associated with cytomegalovirus viremia in renal allografts. N Engl J Med. 1981;305:57-63.

115. Smith JM, Dharnidharka VR. Viral surveillance and subclinical viral infection in pediatric kidney transplantation. Pediatr Nephrol (Berlin, Germany). 2014. published on line. doi: 10.1007/s00467-014-2866-8

116. Onuigbo M, Haririan A, Ramos E, Klassen D, Wali R, Drachenberg C. Cytomegalovirusinduced glomerular vasculopathy in renal allografts: a report of two cases. Am J Transplant. 2002;2:684-8.

117. Ross SA, Novak Z, Pati S, Boppana SB. Overview of the diagnosis of cytomegalovirus infection. Infect Disord Drug Targets. 2011;11:466-74.

118. Hodson EM, Craig JC, Strippoli GF, Webster AC. Antiviral medications for preventing cytomegalovirus disease in solid organ transplant recipients. Cochrane Database Syst Rev. 2008;16:CD003774.

119. Alexander BT, Hladnik LM, Augustin KM, Casabar E, McKinnon PS, Reichley RM, Ritchie DJ, Westervelt P, Dubberke ER. Use of cytomegalovirus intravenous immune globulin for the adjunctive treatment of cytomegalovirus in hematopoietic stem cell transplant recipients. Pharmacotherapy. 2010;30:554-61.

120. Lin CY, Hsu HC, Hung HY. Nephrotic syndrome associated with varicella infection. Pediatrics. 1985;75:1127-31.

121. Minkowitz S, Wenk R, Friedman E, Yuceoglu A, Berkovich S. Acute glomerulonephritis associated with varicella infection. Am J Med. 1968;44:489-92.

122. Cataudella JA, Young ID, Iliescu EA. Epstein-Barr virus-associated acute interstitial nephritis: infection or immunologic phenomenon? Nephron. 2002;92:437-9.

123. Dylewski J, Roy I, Eid J. Acute renal failure associated with acute Epstein-Barr virus infection. Infect Dis Clin Pract. 2008;16:127-8.

124. Verma N, Arunabh S, Brady TM, Charytan C. Acute interstitial nephritis secondary to infectious mononucleosis. Clin Nephrol. 2002;58:151-4.

125. Okada H, Ikeda N, Kobayashi T, Inoue T, Kanno Y, Sugahara S, Nakamoto H, Yamamoto T, Suzuki H. An atypical pattern of Epstein-Barr virus infection in a case with idiopathic tubulointerstitial nephritis. Nephron. 2002;92:440-4.

126. Bhimma R, Purswani MU, Kala U. Kidney disease in children and adolescents with perinatal HIV-1 infection. J Int AIDS Soc. 2013;16:18596.

127. McCulloch MI, Ray PE. Kidney disease in HIV-positive children. Semin Nephrol. 2008;28:585-94. 
128. Ray PE, Rakusan T, Loechelt BJ, Selby DM, Liu XH, Chandra RS. Human immunodeficiency virus (HIV)-associated nephropathy in children from the Washington, D.C. area: 12 years' experience. Semin Nephrol. 1998;18:396-405.

129. Wyatt CM, Klotman PE. HIV-1 and HIV-associated nephropathy 25 years later. Clin J Am Soc Nephrol. 2007;2:S20-4.

130. Nebuloni M, Tosoni A, Boldorini R, Monga G, Carsana L, Bonetto S, Abeli C, Caldarelli R, Vago L, Costanzi G. BK virus renal infection in a patient with the acquired immunodeficiency syndrome. Arch Pathol Lab Med. 1999;123:807-11.

131. Kopp JB, Falloon J, Filie A, Abati A, King C, Hortin GL, Mican JM, Vaughan E, Miller $\mathrm{KD}$. Indinavir-associated interstitial nephritis and urothelial inflammation: clinical and cytologic findings. Clin Infect Dis. 2002;34:1122-8.

132. Dimitrakopoulos AN, Kordossis T, Hatzakis A, Moutsopoulos HM. Mixed cryoglobulinemia in HIV-1 infection: the role of HIV-1. Ann Intern Med. 1999;130:226-30.

133. Turner ME, Kher K, Rakusan T, D’Angelo L, Kapur S, Selby D, Ray PE. A typical hemolytic uremic syndrome in human immunodeficiency virus-1-infected children. Pediatr Nephrol. 1997;11:161-3.

134. Winston JA, Klotman ME, Klotman PE. HIV-associated nephropathy is a late, not early, manifestation of HIV-1 infection. Kidney Int. 1999;55:1036-40.

135. Medapalli RK, He JC, Klotman PE. HIV-associated nephropathy: pathogenesis. Curr Opin Nephrol Hypertens. 2011;20:306-11.

136. D'Agati V, Appel GB. Renal pathology of human immunodeficiency virus infection. Semin Nephrol. 1998;18:406-21.

137. Wyatt CM, Klotman PE, D'Agati VD. HIV-associated nephropathy: clinical presentation, pathology, and epidemiology in the era of antiretroviral therapy. Semin Nephrol. 2008;28:513-22.

138. Winston JA, Bruggeman LA, Ross MD, Jacobson J, Ross L, D’Agati VD, Klotman PE, Klotman ME. Nephropathy and establishment of a renal reservoir of HIV type 1 during primary infection. N Engl J Med. 2001;344:1979-84.

139. Marras D, Bruggeman LA, Gao F, Tanji N, Mansukhani MM, Cara A, Ross MD, Gusella GL, Benson G, D'Agati VD, et al. Replication and compartmentalization of HIV-1 in kidney epithelium of patients with HIV-associated nephropathy. Nat Med. 2002;8:522-6.

140. Jao J, Wyatt CM. Antiretroviral medications: adverse effects on the kidney. Adv Chronic Kidney Dis. 2010;17:72-82.

141. Fernandez-Fernandez B, Montoya-Ferrer A, Sanz AB, Sanchez-Nino MD, Izquierdo MC, Poveda J, Sainz-Prestel V, Ortiz-Martin N, Parra-Rodriguez A, Selgas R, et al. Tenofovir nephrotoxicity: 2011 update. AIDS Res Treat. 2011;2011:354908.

142. Perazella MA. Tenofovir-induced kidney disease: an acquired renal tubular mitochondriopathy. Kidney Int. 2010;78:1060-3.

143. Ingulli E, Tejani A, Fikrig S, Nicastri A, Chen CK, Pomrantz A. Nephrotic syndrome associated with acquired immunodeficiency syndrome in children. J Pediatr. 1991;119:710-6.

144. Morales E, Martinez A, Sanchez-Ayuso J, Gutierrez E, Mateo S, Martinez MA, Herrero JC, Praga M. Spontaneous improvement of the renal function in a patient with HIV-associated focal glomerulosclerosis. Am J Nephrol. 2002;22:369-71.

145. Yamamoto T, Noble NA, Miller DE, Gold LI, Hishida A, Nagase M, Cohen AH, Border WA. Increased levels of transforming growth factor-beta in HIV-associated nephropathy. Kidney Int. 1999;55:579-92. 
146. Ross MJ, Fan C, Ross MD, Chu T-H, Shi Y, Kaufman L, Zhang W, Klotman ME, Klotman PE. HIV-1 infection initiates an inflammatory cascade in human renal tubular epithelial cells. J Acquir Immune Defic Syndr. 2006;42:1-11.

147. Abbott KC, Trespalacios FC, Agodoa LY, Ahuja TS. HIVAN and medication use in chronic dialysis patients in the United States: analysis of the USRDS DMMS Wave 2 study. BMC Nephrol. 2003;4:5.

148. Kimmel PL, Bosch JP, Vassalotti JA. Treatment of human immunodeficiency virus (HIV)associated nephropathy. Semin Nephrol. 1998;18:446-58.

149. Gruber SA, Doshi MD, Cincotta E, Brown KL, Singh A, Morawski K, Alangaden G, Chandrasekar P, Losanoff JE, West MS, et al. Preliminary experience with renal transplantation in HIV + recipients: low acute rejection and infection rates. Transplantation. 2008;86:269-74.

150. Kumar MS, Sierka DR, Damask AM, Fyfe B, McAlack RF, Heifets M, Moritz MJ, Alvarez D, Kumar A. Safety and success of kidney transplantation and concomitant immunosuppression in HIV-positive patients. Kidney Int. 2005;67:1622-9.

151. Pinto M, Dobson S. BK and JC virus: a review. J Infect. 2014;68 Suppl 1:S2-8.

152. Drachenberg CB, Papadimitriou JC. Polyomavirus-associated nephropathy: update in diagnosis. Transpl Infect Dis. 2006;8:68-75.

153. Liptak P, Kemeny E, Ivanyi B. Primer: histopathology of polyomavirus-associated nephropathy in renal allografts. Nat Clin Pract Nephrol. 2006;2:631-6.

154. Barraclough KA, Isbel NM, Staatz CE, Johnson DW. BK virus in kidney transplant recipients: the influence of immunosuppression. J Transplant. 2011;2011:750836.

155. Acott PD, Hirsch HH. BK virus infection, replication, and diseases in pediatric kidney transplantation. Pediatr Nephrol. 2007;22:1243-50.

156. Lin PL, Vats AN, Green M. BK virus infection in renal transplant recipients. Pediatr Transplant. 2001;5:398-405.

157. van Aalderen MC, Heutinck KM, Huisman C, ten Berge IJ. BK virus infection in transplant recipients: clinical manifestations, treatment options and the immune response. Neth $\mathrm{J}$ Med. 2012;70:172-83.

158. Emerson LL, Carney HM, Layfield LJ, Sherbotie JR. Collecting duct carcinoma arising in association with BK nephropathy post-transplantation in a pediatric patient. A case report with immunohistochemical and in situ hybridization study. Pediatr Transpl. 2008;12:600-5.

159. Kausman JY. Association of renal adenocarcinoma and BK virus nephropathy posttransplantation. Pediatr Nephrol. 2004;19:459.

160. Ramos E, Drachenberg CB, Papadimitriou JC, Hamze O, Fink JC, Klassen DK, Drachenberg $\mathrm{RC}$, Wiland A, Wali R, Cangro $\mathrm{CB}$, et al. Clinical course of polyomavirus nephropathy in 67 renal transplant patients. J Am Soc Nephrol. 2002;13:2145-51.

161. Johnston O, Jaswal D, Gill JS, Doucette S, Fergusson DA, Knoll GA. Treatment of polyomavirus infection in kidney transplant recipients: a systematic review. Transplantation. 2010;89:1057-70.

162. Jeffs B. A clinical guide to viral haemorrhagic fevers: Ebola, Marburg and Lassa. Trop Doct. 2006;36:1-4.

163. Elling R, Henneke P, Hatz C, Hufnagel M. Dengue fever in children: where are we now? Pediatr Infect Dis J. 2013;32:1020-2.

164. Boonpucknavig V, Bhamarapravati N, Boonpucknavig S, Futrakul P, Tanpaichitr P. Glomerular changes in dengue hemorrhagic fever. Arch Pathol Lab Med. 1976;100:206-12. 
165. Molyneux EM, Maitland K. Intravenous fluids-getting the balance right. N Engl J Med. 2005;353:941-4.

166. Wills BA, Nguyen MD, Ha TL, Dong TH, Tran TN, Le TT, Tran VD, Nguyen TH, Nguyen VC, Stepniewska K, et al. Comparison of three fluid solutions for resuscitation in dengue shock syndrome. N Engl J Med. 2005;353:877-89.

167. Appannanavar SB, Mishra B. An update on Crimean Congo hemorrhagic fever. J Global Infect Dis. 2011;3:285-92.

168. Soares-Weiser K, Thomas S, Thomson G, Garner P. Ribavirin for Crimean-Congo hemorrhagic fever: systematic review and meta-analysis. BMC Infect Dis. 2010;10:207.

169. Watson DC, Sargianou M, Papa A, Chra P, Starakis I, Panos G. Epidemiology of Hantavirus infections in humans: a comprehensive, global overview. Crit Rev Microbiol. 2014;40:261-72.

170. Manigold T, Vial P. Human hantavirus infections: epidemiology, clinical features, pathogenesis and immunology. Swiss Med Wkly. 2014;144:w13937.

171. Grcevska L, Polenakovic M, Oncevski A, Zografski D, Gligic A. Different pathohistological presentations of acute renal involvement in Hantaan virus infection: report of two cases. Clin Nephrol. 1990;34:197-201.

172. Rusnak JM, Byrne WR, Chung KN, Gibbs PH, Kim TT, Boudreau EF, Cosgriff T, Pittman P, Kim KY, Erlichman MS, et al. Experience with intravenous ribavirin in the treatment of hemorrhagic fever with renal syndrome in Korea. Antiviral Res. 2009;81:68-76.

173. Allen CW, Alexander SI. Adenovirus associated haematuria. Arch Dis Child. 2005;90:305-6.

174. Storsley L, Gibson IW. Adenovirus interstitial nephritis and rejection in an allograft. J Am Soc Nephrol. 2011;22:1423-7.

175. Parasuraman R, Zhang PL, Samarapungavan D, Rocher L, Koffron A. Severe necrotizing adenovirus tubulointerstitial nephritis in a kidney transplant recipient. Case Rep Transplant. 2013;2013:969186.

176. Pasch A, Frey FJ. Coxsackie B viruses and the kidney-a neglected topic. Nephrol Dial Transplant. 2006;21:1184-7.

177. Beigel J, Bray M. Current and future antiviral therapy of severe seasonal and avian influenza. Antiviral Res. 2008;78:91-102.

178. Muthuri SG, Venkatesan S, Myles PR, Leonardi-Bee J, Al Khuwaitir TS, Al Mamun A, Anovadiya AP, Azziz-Baumgartner E, Baez C, Bassetti M, et al. Effectiveness of neuraminidase inhibitors in reducing mortality in patients admitted to hospital with influenza A H1N1pdm09 virus infection: a meta-analysis of individual participant data. Lancet Respir Med. 2014;2:395-404.

179. Lin CY, Hsu HC. Measles and acute glomerulonephritis. Pediatrics. 1983;71:398-401.

180. Kabakus N, Aydinoglu H, Bakkaloglu SA, Yekeler H. Mumps interstitial nephritis: a case report. Pediatr Nephrol. 1999;13:930-1.

181. Lin CY, Chen WP, Chiang H. Mumps associated with nephritis. Child Nephrol Urol. 1990;10:68-71.

182. Lau YL, Peiris JSM. Pathogenesis of severe acute respiratory syndrome. Curr Opin Immunol. 2005;17:404-10.

183. Chu KH, Tsang WK, Tang CS, Lam MF, Lai FM, To KF, Fung KS, Tang HL, Yan WW, Chan $\mathrm{HW}$, et al. Acute renal impairment in coronavirus-associated severe acute respiratory syndrome. Kidney Int. 2005;67:698-705. 
184. Farcas GA, Poutanen SM, Mazzulli T, Willey BM, Butany J, Asa SL, Faure P, Akhavan P, Low DE, Kain KC. Fatal severe acute respiratory syndrome is associated with multiorgan involvement by coronavirus. J Infect Dis. 2005;191:193-7.

185. Ding Y, He L, Zhang Q, Huang Z, Che X, Hou J, Wang H, Shen H, Qiu L, Li Z, et al. Organ distribution of severe acute respiratory syndrome (SARS) associated coronavirus (SARS$\mathrm{CoV}$ ) in SARS patients: implications for pathogenesis and virus transmission pathways. J Pathol. 2004;203:622-30.

186. Li W, Moore MJ, Vasilieva N, Sui J, Wong SK, Berne MA, Somasundaran M, Sullivan JL, Luzuriaga K, Greenough TC, et al. Angiotensin-converting enzyme 2 is a functional receptor for the SARS coronavirus. Nature. 2003;426:450-4.

187. Eckerle I, Muller MA, Kallies S, Gotthardt DN, Drosten C. In-vitro renal epithelial cell infection reveals a viral kidney tropism as a potential mechanism for acute renal failure during Middle East Respiratory Syndrome (MERS) coronavirus infection. Virol J. 2013;10:359.

188. Giglioli G. Malaria and renal disease, with special reference to British Guiana. II The effect of malaria eradication on the incidence of renal disease in British Guiana. Ann Trop Med Parasitol. 1962;56:225-41.

189. Hendrickse RG, Gilles HM. The nephrotic syndrome and other renal diseases in children in Western Nigeria. East Afr Med J. 1963;40:186-201.

190. Ehrich JHH, Eke FU. Malaria-induced renal damage: facts and myths. Pediatr Nephrol. 2007;22:626-37.

191. Boonpucknavig V, Sitprija V. Renal disease in acute Plasmodium falciparum infection in man. Kidney Int. 1979;16:44-52.

192. Elsheikha HM, Sheashaa HA. Epidemiology, pathophysiology, management and outcome of renal dysfunction associated with plasmodia infection. Parasitol Res. 2007;101:1183-90.

193. Maitland K, Levin M, English M, Mithwani S, Peshu N, Marsh K, Newton CRJC. Severe P. falciparum malaria in Kenyan children: evidence for hypovolaemia. QJM. 2003;96:427-34.

194. Maitland K, Pamba A, Newton CRJC, Levin M. Response to volume resuscitation in children with severe malaria. Pediatr Crit Care Med. 2003;4:426-31.

195. Maitland K, Kiguli S, Opoka RO, Engoru C, Olupot-Olupot P, Akech SO, Nyeko R, Mtove G, Reyburn H, Lang T, et al. Mortality after fluid bolus in African children with severe infection. N Engl J Med. 2011;364:2483-95.

196. Van den Ende J, Coppens G, Verstraeten T, Van Haegenborgh T, Depraetere K, Van Gompel A, Van den Enden E, Clerinx J, Colebunders R, Peetermans WE, et al. Recurrence of blackwater fever: triggering of relapses by different antimalarials. Trop Med Int Health. 1998;3:632-9.

197. Barsoum RS. Schistosomiasis and the kidney. Semin Nephrol. 2003;23:34-41.

198. Seck SM, Sarr ML, Dial MC, Ka EF. Schistosoma haematobium-associated glomerulopathy. Indian J Nephrol. 2011;21:201-3.

199. van Griensven J, Diro E. Visceral leishmaniasis. Infect Dis Clin North Am. 2012;26:309-22.

200. Caravaca F, Munoz A, Pizarro JL, Saez de Santamaria J, Fernandez-Alonso J. Acute renal failure in visceral leishmaniasis. Am J Nephrol. 1991;11:350-2.

201. Yap HK, Woo KT, Yeo PP, Chiang GS, Singh M, Lim CH. The nephrotic syndrome associated with filariasis. Ann Acad Med Singapore. 1982;11:60-3.

202. Graziani G, Cucchiari D, Verdesca S, Balzarini L, Montanelli A, Ponticelli C. Chyluria associated with nephrotic-range proteinuria: pathophysiology, clinical picture and therapeutic options. Nephron Clin Pract. 2011;119:c248-53; discussion c254.

203. Moro P, Schantz PM. Echinococcosis: a review. Int J Infect Dis. 2009;13:125-33. 
204. Gelman R, Brook G, Green J, Ben-Itzhak O, Nakhoul F. Minimal change glomerulonephritis associated with hydatid disease. Clin Nephrol. 2000;53:152-5.

205. Kauffman CA. Diagnosis and management of fungal urinary tract infection. Infect Dis Clin North Am. 2014;28:61-74.

206. Stocker M, Caduff JH, Spalinger J, Berger TM. Successful treatment of bilateral renal fungal balls with liposomal amphotericin B and fluconazole in an extremely low birth weight infant. Eur J Pediatr. 2000;159:676-8.

207. Levin M, Hjelm M, Kay JD, Pincott JR, Gould JD, Dinwiddie R, Matthew DJ. Haemorrhagic shock and encephalopathy: a new syndrome with a high mortality in young children. Lancet. 1983;2:64-7.

208. Levin M, Pincott JR, Hjelm M, Taylor F, Kay J, Holzel H, Dinwiddie R, Matthew DJ. Hemorrhagic shock and encephalopathy: clinical, pathologic, and biochemical features. J Pediatr. 1989;114:194-203.

209. Eleftheriou D, Levin M, Shingadia D, Tulloh R, Klein NJ, Brogan PA. Management of Kawasaki disease. Arch Dis Child. 2014;99:74-83.

210. Watanabe T. Kidney and urinary tract involvement in Kawasaki disease. Int $\mathrm{J}$ Pediatr. 2013;2013:831834.

211. Chaudhary R, Singh K, Jain N, Biswas R. Chronic flank pain, fever and an unusual diagnosis. BMJ Case Rep. 2011;2011. published online. doi: 10.1136/bcr.08.2011.4646.

212. Gupta G, Singh R, Kotasthane DS, Kotasthane VD, Kumar S. Xanthogranulomatous pyelonephritis in a male child with renal vein thrombus extending into the inferior vena cava: a case report. BMC Pediatr. 2010;10:47.

213. Schildgen V, van den Hoogen B, Fouchier R, Tripp RA, Alvarez R, Manoha C, Williams J, Schildgen O. Human Metapneumovirus: lessons learned over the first decade. Clinical Microbiology Reviews. 2011;24(4):734-754.

214. Allander T et al. Cloning of a human parvovirus by molecular screening of respiratory tract samples. PNAS. 2005;102(34):12891-6. 